\title{
On the Extension of Multidimensional Speckle Noise Model From Single-Look to Multilook SAR Imagery
}

\author{
Carlos López-Martínez, Member, IEEE, and Eric Pottier, Member, IEEE
}

\begin{abstract}
Speckle noise represents one of the major problems when synthetic aperture radar (SAR) data are considered. Despite the fact that speckle is caused by the scattering process itself, it must be considered as a noise source due to the complexity of the scattering process. The presence of speckle makes data interpretation difficult, but it also affects the quantitative retrieval of physical parameters. In the case of one-dimensional SAR systems, speckle is completely determined by a multiplicative noise component. Nevertheless, for multidimensional SAR systems, speckle results from the combination of multiplicative and additive noise components. This model has been first developed for single-look data. The objective of this paper is to extend the single-look data model to define a multilook multidimensional speckle noise model. The asymptotic analysis of this extension, for a large number of averaged samples, is also considered to assess the model properties. Details and validation of the multilook multidimensional speckle noise model are provided both theoretically and by means of experimental SAR data acquired by the experimental synthetic aperture radar system, operated by the German Aerospace Center.
\end{abstract}

Index Terms-Covariance matrix, interferometry, multidimensional signal, noise modeling, polarimetry, quantitative remote sensing, speckle noise, synthetic aperture radar (SAR).

\section{INTRODUCTION}

$\mathbf{S}$ YNTHETIC aperture radars (SARs) have been successfully employed to observe, study, and characterize the Earth's surface [1]. Among the diverse features of these systems, it is of importance to mention the independence of their imaging capabilities from weather conditions and from the day-night cycle. Nevertheless, the importance of SAR systems lies essentially on the potential to retrieve Earthsurface information with a high spatial resolution. The dimension perpendicular to the platform movement is referred to as range. In range, spatial resolution is achieved by classical radar techniques based on the use of a large signal bandwidth along with matched filtering techniques [1]. In azimuth, the spatial resolution is obtained by means of the synthesis of a very large virtual antenna, exploiting the Doppler effect caused by the relative motion of the SAR system with respect to a target of reference. The analysis of the Doppler effect needs a coherent recording and processing of the scattered radar echoes, producing, as a consequence, complex reflectivity images. This

Manuscript received July 4, 2006; revised September 27, 2006.

C. López-Martínez is with the Signal Theory and Communications Department, Universitat Politècnica de Catalunya, 08034 Barcelona, Spain.

E. Pottier is with the Institute of Electronics and Telecommunications of Rennes, SAPHIR Team, Universite de Rennes 1, 35042 Rennes Cedex, France.

Digital Object Identifier 10.1109/TGRS.2006.887012 complex nature of SAR systems also yields speckle noise. The scattering process itself produces speckle, but the complexity of this scattering process makes it necessary to consider it as a noise component corrupting the useful information contained in the scattered signal [2], [3]. For one-dimensional SAR systems, speckle is characterized by a multiplicative noise component [2]-[5].

One-dimensional SAR systems have evolved in the last decade toward multidimensional configurations, making quantitative remote sensing possible. Among the different possibilities, the exploitation of the space or the polarimetric diversities, giving rise to interferometric (InSAR) [6], [7], and polarimetric (PolSAR) [8] systems, respectively, is the most employed scheme. Multidimensional SAR systems are also affected by speckle. However, it does not only affect SAR images but also the correlation structure that characterizes the ensemble of SAR images. In this case, speckle is due to the combination of multiplicative and additive noise components, which is determined by the correlation structure of the data [9]. As shown in [9], this model was able to describe speckle noise for single-look multidimensional SAR data. This paper aims to extend this single-look multidimensional speckle noise model to multilook data. The availability of the single-look multidimensional noise model and, specially, the identification of the complex additive speckle noise component made possible the determination of the importance of this noise source in coherence estimation, as it is on the origin of the coherence bias for low coherences [10]. This model has also allowed to show that, when considering PolSAR data, this additive speckle noise source is on the origin of the biases associated to the information extracted by means of those target decomposition theorems based on the eigen decomposition of the covariance or coherency matrices [11], [12]. There exist several arguments which justify the extension to the multilook SAR data case. The first one is found in the fact that, sometimes, multidimensional SAR data are only delivered in multilook format. The second motive supporting such an extension is the necessity to determine the effects of speckle noise upon the quantitative estimation of the physical information from the data. From a wider point of view, an extension could be employed to determine the effect of any multidimensional speckle filtering algorithm on the information estimation process.

This paper is divided into four main sections. Section II introduces the necessary mathematical framework to analyze the problem of characterizing multilook multidimensional speckle. Section III concentrates on the study of the multilook multidimensional noise problem. Section III-A is devoted to derive a multilook speckle noise model valid for interferometric applications, which is employed to derive the multilook model 
in Section III-B, which is valid for any number of averaged samples. An asymptotic analysis of the model for a large number of averaged samples is also the object of this paper. Section IV introduces a theoretical interpretation of the multilook multidimensional speckle noise model, which is validated on the experimental PolSAR data in Section V. Finally, Section VI contains the main conclusion of this paper.

\section{STATISTICAL CHARACTERIZATION OF Multidimensional SAR DATA}

A multidimensional SAR system delivers a set of $m$ SAR images represented, for every data pixel, by the target vector [14]

$$
\mathbf{k}=\left[S_{1}, S_{2}, \ldots, S_{m}\right]^{\mathrm{T}}
$$

where $S_{k}$, for $k=1,2, \ldots, m$, represents each one of the complex SAR images and ${ }^{\mathrm{T}}$ denotes the vector transposition. Single baseline InSAR and along-track interferometry data are characterized by $m=2$; PolSAR data present a dimensionality equal to $m=3$ or $m=4$, depending whether the data are acquired in a monostatic or in a bistatic configuration, respectively. Under these same conditions, polarimetric interferometric SAR data (PolInSAR) can be characterized by $m=6$ or $m=8$. Higher dimensional SAR systems may be considered under multibaseline [15], multifrequency [16], [17], or even multitime acquisition schemes. In those cases in which the scattering process is due to a point scattering mechanism, $\mathrm{k}$ represents an $m$-dimensional deterministic variable [18]. However, when $\mathbf{k}$ represents a distributed scattering mechanism, it must be considered as an $m$-dimensional random vector [19], [20]. In coherent imaging techniques, this unpredictable nature receives the name of speckle noise [1], [4], [21].

The Earth's surface presents a nonhomogeneous nature that is translated to the SAR data. This wide diversity of possible scenarios implies that the statistical behavior of $\mathbf{k}$, in a general case, cannot be embedded into a single multidimensional probability density function (pdf). Thus, in order to derive a simple mathematical model, certain restrictions and simplifications must be imposed in the scattering process resulting in $\mathbf{k}$. Therefore, a resolution cell of a distributed scatterer is modeled as a set of elementary or point scatterers. Then, the scattered wave emanating from a resolution cell is assumed to be the linear superposition of the individual responses of the elementary point scatterers. Under the hypothesis that the amplitude and the phase of every elementary wave are mutually uncorrelated, but also uncorrelated with all other elementary waves, and the premise that these elementary phases are uniformly distributed, the real and imaginary parts of every particular SAR image $S_{k}$ for $k=1,2, \ldots, m$ may be statistically characterized, on the basis of the Central Limit Theorem, by uncorrelated zeromean Gaussian pdfs [2]. Thus, the vector $\mathbf{k}$ may be characterized by a zero-mean multidimensional complex Gaussian pdf [22], [23]

$$
p_{\mathbf{k}}(\mathbf{k})=\frac{1}{\pi^{m}|\mathbf{C}|} \exp \left(-\mathbf{k}^{\mathrm{H}} \mathbf{C}^{-1} \mathbf{k}\right)
$$

where ${ }^{\mathrm{H}}$ refers to the transpose complex conjugation, $|\mathbf{X}|$ is the determinant of the matrix $\mathbf{X}$, and $\mathbf{C}$ consists of the $m \times m$ semipositive semidefinite Hermitian covariance matrix

$$
\begin{aligned}
\mathbf{C} & =E\left\{\mathbf{k k}^{\mathrm{H}}\right\} \\
& =\left[\begin{array}{cccc}
E\left\{S_{1} S_{1}^{\mathrm{H}}\right\} & E\left\{S_{1} S_{2}^{\mathrm{H}}\right\} & \cdots & E\left\{S_{1} S_{m}^{\mathrm{H}}\right\} \\
E\left\{S_{2} S_{1}^{\mathrm{H}}\right\} & E\left\{S_{2} S_{2}^{\mathrm{H}}\right\} & \cdots & E\left\{S_{2} S_{m}^{\mathrm{H}}\right\} \\
\vdots & \vdots & \ddots & \vdots \\
E\left\{S_{m} S_{1}^{\mathrm{H}}\right\} & E\left\{S_{m} S_{2}^{\mathrm{H}}\right\} & \cdots & E\left\{S_{m} S_{m}^{\mathrm{H}}\right\}
\end{array}\right] .
\end{aligned}
$$

Since $\mathbf{k}$ is a zero-mean vector, the distribution presented in (2) is completely determined by $\mathbf{C}$, which contains all the necessary information to characterize $\mathbf{k}$.

The Gaussian hypothesis (2) is unable to characterize complex scenarios such as highly textured or urban areas [21], where, among others, the multidimensional $K$-distribution model has demonstrated to be able to accommodate data randomness. These more elaborated models are based on increasing the number of parameters necessary to describe the data variability and on presenting more complex analytical expressions to describe the data pdf. Although the Gaussian scattering model has been shown to be valid in describing the data resulting from a homogeneous clutter, its usefulness has also been demonstrated in the diverse fields of SAR data processing: data analysis [10], [12], [24], speckle filtering [25], [26], or PolSAR data classification [27]-[29]. In addition, despite its limitations in front of more complex distribution models, the hypothesis of Gaussian distributed SAR images allowed the analytical analysis of the data.

As $\mathbf{k}$ is a zero-mean vector, data characterization must be performed by means of higher order moments. Consequently, in the case of Gaussian scattering, this process is reduced to estimate $\mathbf{C}$. Under the hypothesis that the processes of $\mathbf{k}$ are stationary, jointly stationary, and also ergodic (in mean), the ensemble averages in (3) can be substituted with spatial averages, resulting in the so-called sample covariance matrix

$$
\mathbf{Z}_{n}=\left\langle\mathbf{k k}^{\mathrm{H}}\right\rangle_{n}=\frac{1}{n} \sum_{i=1}^{n} \mathbf{k}_{i} \mathbf{k}_{i}^{\mathrm{H}}
$$

where $n$ represents the number of samples, also called looks, employed to estimate $\mathbf{C}$ and the symbol $\langle\cdot\rangle_{n}$ denotes the spatial average. The sample covariance matrix $\mathbf{Z}_{n}$ is statistically determined by the Wishart pdf [13], [22], [23]

$$
p_{\mathbf{Z}_{n}}\left(\mathbf{Z}_{n}\right)=\frac{n^{m n}\left|\mathbf{Z}_{n}\right|^{n-m}}{|\mathbf{C}|^{n} \tilde{\Gamma}_{m}(n)} \operatorname{etr}\left(-n \mathbf{C}^{-1} \mathbf{Z}_{n}\right)
$$

where $\operatorname{etr}(\mathbf{X})$ is the exponential of the trace of the matrix $\mathbf{X}$ and the multivariate gamma function is defined as follows

$$
\tilde{\Gamma}_{m}(n)=\pi^{m(m-1) / 2} \prod_{i=1}^{m} \Gamma(n-i+1)
$$

where $\Gamma(x)$ is the gamma function. The Wishart pdf presented in (5) is only valid for $n \geq m$. As observed in (2) and (5), the Gaussian and the Wishart pdfs are determined by the covariance matrix $\mathbf{C}$. Hence, only second-order moments are necessary for a complete characterization of multidimensional SAR data. A very well-known result, in the case of the multivariate Gaussian 
pdf, states that a $p$ th-order central product moment is zero if $p$ is odd and is equal to a sum of the products of the covariances when $p$ is even [30]. A generalization of this result shows that the $p$ th-order moments of the sample covariance matrix $E\left\{\mathbf{Z}_{n}^{p}\right\}$ are only a function of $\mathbf{C}[31]$.

\section{A. Single-Look Multidimensional Speckle Noise Model}

The covariance matrix estimation process is of central importance in multidimensional SAR imagery. In the remote sensing literature, this process has always been considered from the optic of multidimensional speckle noise reduction, with an interest almost exclusively focused on PolSAR systems. Among the vast literature on this subject, it is important to notice, for instance, the works of Lopes and coauthors [5], [24], Shou and Skriver [25], and others. In particular, the work developed by Lee et al. [26] represents a cornerstone in polarimetric speckle noise filtering. Nevertheless, all these approaches present a key lack since, as mentioned, they are based on considering the multidimensional speckle noise problem just from the filtering point of view, neglecting somehow the modeling and the study of the effects of the multidimensional speckle noise problem. In [26], Lee et al. presented a first attempt to determine a multidimensional speckle noise model, concluding that multidimensional speckle could not be modeled by a completely additive or multiplicative noise source.

Since $\mathbf{C}$ must be estimated from a limited number of samples, this estimation shall present an error due to the presence of speckle. One way to consider it is to think of the error as being some kind of noise. This alternative has been first considered and validated by the study in [9], resulting in the single-look multidimensional speckle noise model. This model was constructed based on the observation that all the covariance matrix entries consist of the Hermitian product of a couple of SAR images. As detailed in [9], the one-look Hermitian product of two SAR images may be modeled as follows:

$$
\begin{aligned}
S_{i} S_{j}^{*}=\psi \bar{z}_{n} n_{m} N_{c} \exp \left(j \phi_{x}\right) & \\
& +\psi\left(|\rho|-N_{c} \bar{z}_{n}\right) \exp \left(j \phi_{x}\right)+\psi\left(n_{\mathrm{ar}}+j n_{\mathrm{ai}}\right)
\end{aligned}
$$

where $S_{i} S_{j}^{*}$, for $i, j=1,2, \ldots, m$, represents the Hermitian product of any two elements of $\mathbf{k}$. The parameters $\psi, \bar{z}_{n}, N_{c}$, and $\phi_{x}$ depend on the particular Hermitian product $S_{i} S_{j}^{*}$ for $i, j=1,2, \ldots, m$, in which precise definitions are presented afterward in the text or they may be found in [9]. In (7), the term $\psi|\rho| \exp \left(j \phi_{x}\right)$ represents the useful signal component that is contaminated by speckle. As one may observe, speckle is characterized by two noise components. On the one hand, a homogeneous real multiplicative noise source is denoted by $n_{m}$ and characterized by

$$
E\left\{n_{m}\right\}=1 \quad \operatorname{var}\left\{n_{m}\right\}=1 .
$$

The second speckle noise component is a nonhomogeneous complex additive noise source denoted by $n_{\text {ar }}+j n_{\text {ai }}$ such that

$E\left\{n_{\mathrm{ar}}\right\}=E\left\{n_{\mathrm{ai}}\right\}=1 \quad \operatorname{var}\left\{n_{\mathrm{ar}}\right\}=\operatorname{var}\left\{n_{\mathrm{ai}}\right\}=\frac{1}{2}\left(1-|\rho|^{2}\right)^{1.32}$.

The combination of these noise sources is determined by the complex correlation coefficient which characterizes the
Hermitian product $S_{i} S_{j}^{*}$ for $i, j=1,2, \ldots, m$. For instance, for $i=j$, i.e., $\rho=1 \exp (j 0)$, (7) reduces to the classical multiplicative speckle noise model employed to describe the intensity of a particular SAR image.

The multiplicative speckle noise component can be transformed straightforwardly into an additive noise such that

$$
\begin{aligned}
S_{i} S_{j}^{*}= & \psi|\rho| \exp \left(j \phi_{x}\right) \\
& +\psi N_{c} \bar{z}_{n}\left(n_{m}-1\right) \exp \left(j \phi_{x}\right)+\psi\left(n_{\mathrm{ar}}+j n_{\mathrm{ai}}\right) .
\end{aligned}
$$

Taking into account (10) for all the elements constituting the single-look sample covariance matrix allows considering this matrix as

$$
\left.\mathbf{Z}_{n}\right|_{n=1}=\mathbf{C}+\mathbf{N}_{m}+\mathbf{N}_{a}
$$

The matrix $\mathbf{N}_{m}$, that represents the multiplicative components of speckle, is constructed by considering the second additive term of (10) for every element of the sample covariance matrix. The matrix $\mathbf{N}_{a}$, that accounts for the additive speckle noise components, is equally constructed, but by means of the third additive term of (10). $\mathbf{N}_{a}$ presents the particularity that its diagonal elements are equal to zero. The multidimensional speckle noise model (11) is coherent with the previous models for the covariance matrix employed in the classical radar literature [32]. Nevertheless, (11) extends the model presented in [32], in such a way that it is able to identify a particular structure on the noise, resulting into the multiplicative and additive speckle noise components.

\section{Multilook Multidimensional SPECKLE NOISE MODEL}

The main parameter characterizing the Hermitian product of two SAR images $S_{i} S_{j}^{*}$, for $i, j=1,2, \ldots, m$, is the complex correlation coefficient

$\rho=|\rho| \exp \left(j \phi_{x}\right)=\frac{E\left\{S_{i} S_{j}^{*}\right\}}{\sqrt{E\left\{\left|S_{i}\right|^{2}\right\} E\left\{\left|S_{j}\right|^{2}\right\}}}, \quad i, j=1,2, \ldots, m$

where $|\rho|$ denotes the coherence and $\phi_{x}$ represents the average phase information. The average power in the two channels is defined as $\psi=\left(E\left\{\left|S_{i}\right|^{2}\right\} E\left\{\left|S_{j}\right|^{2}\right\}\right)^{1 / 2}$. The measured phase of the Hermitian product $S_{i} S_{j}^{*}$, for $i, j=1,2, \ldots, m$, in which the average value is in fact $\phi_{x}$, is of capital importance since its study allowed obtaining of the single-look multidimensional speckle noise model (7). In the following, the same approach presented in [9] is followed, but examining all the aspects which result from considering multilook SAR data.

\section{A. Multilook Phasor Noise Model}

Given the statistical model presented in (2), after considering the averaging process introduced at (4), the phase of the Hermitian product of a pair of SAR images

$$
\left\langle S_{i} S_{j}^{*}\right\rangle_{n}=z \exp (j \phi), \quad i, j=1,2, \ldots, m
$$


is described by the pdf [23], [33]

$p_{\phi}(\phi)=\frac{\Gamma(n+1 / 2)\left(1-|\rho|^{2}\right)^{n} \beta}{2 \sqrt{\pi} \Gamma(n)\left(1-\beta^{2}\right)^{n+1 / 2}}+\frac{\left(1-|\rho|^{2}\right)^{n}}{2 \pi}{ }_{2} F_{1}\left(n, 1 ; \frac{1}{2} ; \beta^{2}\right)$

where $\beta=|\rho| \cos \left(\phi-\phi_{x}\right)$ and ${ }_{2} F_{1}(a, b ; c ; z)$ represents the Gauss hypergeometric function [34], [35]. The behavior of (14) for a large $n$ will be necessary later on for the asymptotic analysis of the multilook speckle noise model considered in this paper. After the calculations are detailed in Appendix A, (14) is reduced to

$p_{\phi}(\phi)=\frac{\beta(1+\operatorname{sgn}(\beta)) \sqrt{n}}{2 \sqrt{\pi}} \frac{\left(1-|\rho|^{2}\right)^{n}}{\left(1-\beta^{2}\right)^{n+1 / 2}}, \quad|\rho| \cos (\phi) \neq 0$

for a large $n$, where $\operatorname{sgn}(x)$ is the sign function. Lee et al. [36] have demonstrated that the phase pdf (14) determines that $\phi$ may be described by the noise model

$$
\phi=\phi_{x}+\nu
$$

where $\phi_{x}$ represents the useful information term and $\nu$ is a zero-mean noise term, in which the variance depends on $n$ and $|\rho|$, but it is independent from $\phi_{x}$. The validity of (16) is restricted to $\left[\phi_{x}-\pi, \phi_{x}+\pi\right)$, since the expectation of $\phi$ does not correspond to $\phi_{x}$ in the interval $[-\pi, \pi)$ [21]. This restriction does not apply when $\phi$ is considered in the complex instead of the real plane. As derived in [9], one can describe the real and imaginary parts of the complex phasor as

$$
\begin{aligned}
& \Re\{\exp (j \phi)\}=N_{c} \cos \left(\phi_{x}\right)+\nu_{1}^{\prime} \cos \left(\phi_{x}\right)-\nu_{2}^{\prime} \sin \left(\phi_{x}\right) \\
& \Im\{\exp (j \phi)\}=N_{c} \sin \left(\phi_{x}\right)+\nu_{1}^{\prime} \sin \left(\phi_{x}\right)+\nu_{2}^{\prime} \cos \left(\phi_{x}\right)
\end{aligned}
$$

where $N_{c}$ is the expected value of $\cos (\nu), \nu_{1}^{\prime}$ and $\nu_{2}^{\prime}$ represent two zero-mean random variables, and $\Re\{z\}$ and $\Im\{z\}$ denote the real and imaginary parts. The combination of these two equations in the complex plane results in the following noise model for the complex phasor $\exp (j \phi)$ :

$$
\exp (j \phi)=\left(N_{c}+\left(\nu_{1}^{\prime}+j \nu_{2}^{\prime}\right)\right) \exp \left(j \phi_{x}\right)
$$

By considering $\phi$ as being characterized by (14), one can find

$$
N_{c}=\frac{\Gamma(n+1 / 2) \Gamma(3 / 2)}{\Gamma(n)}|\rho|_{2} F_{1}\left(\frac{3}{2}-n, \frac{1}{2} ; 2 ;|\rho|^{2}\right)
$$

where the details are in Appendix B. Fig. 1 depicts the evolution of $N_{c}$ as a function of coherence for several values of $n$. The asymptotic behavior of $N_{c}$ for a large $n$ is contemplated in Appendix C. For $|\rho| \neq 0$, the following asymptotic approximation for a large $n$ applies for (20):

$$
N_{c}=1-\frac{1}{8 n}+O\left(\frac{1}{n^{2}}\right)
$$

from where

$$
\lim _{n \rightarrow \infty} N_{c}=1
$$

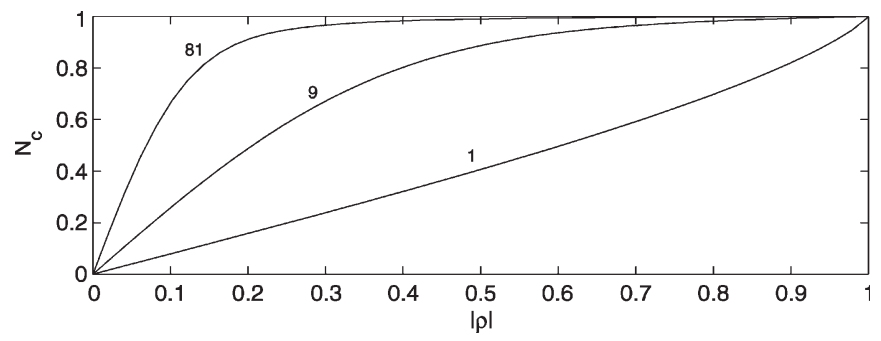

Fig. 1. Parameter $N_{c}$ evaluated for different number of looks $n$.

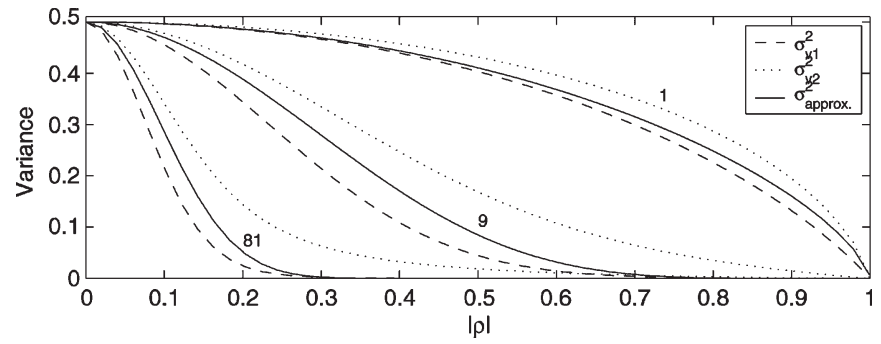

Fig. 2. Variances $\sigma_{\nu_{1}^{\prime}}^{2}$ and $\sigma_{\nu_{2}^{\prime}}^{2}$ evaluated for different number of looks $n$. $\sigma_{\text {approx }}^{2}$ refers here to the approximation introduced in (28). A normalization $\psi=1, \phi_{x}=0$ is considered.

As observed, the limit does not depend on the coherence value. Nevertheless, the point $|\rho|=0$ deserves a special attention since in this case

$$
\lim _{\substack{n \rightarrow \infty \\|\rho|=0}} N_{c}=0
$$

The analysis of the two noise terms in (17) and (18) is now considered. Since by construction both noise sources are zeromean, they must be characterized by means of their variance values. This calculation is detailed in Appendix D, from where it results to

$$
\begin{aligned}
\sigma_{\nu_{1}^{\prime}}^{2} & =\frac{1}{2}\left(1-|\rho|^{2}\right)^{n}{ }_{3} F_{2}\left(\frac{3}{2}, n, 1 ; 2, \frac{1}{2} ;|\rho|^{2}\right)-N_{c}^{2} \\
\sigma_{\nu_{2}^{\prime}}^{2} & =\frac{1}{2}\left(1-|\rho|^{2}\right)^{n}{ }_{3} F_{2}\left(\frac{1}{2}, n, 1 ; 2, \frac{1}{2} ;|\rho|^{2}\right)
\end{aligned}
$$

where ${ }_{3} F_{2}(a, b, c ; d, e ; z)$ is a generalized hypergeometric function [34], [35]. The evaluation of (24) and (25) for different number of looks is depicted in Fig. 2. As observed, an increase of $n$ does not produce the variances $\sigma_{\nu_{1}^{\prime}}^{2}$ and $\sigma_{\nu_{2}^{\prime}}^{2}$ to decrease in the classical form, i.e., $1 / n$, as the phase $\phi$ results from a coherent filtering process, as shown by (4). In the case of the generalized hypergeometric functions ${ }_{3} F_{2}(a, b, c ; d, e ; z)$, as stated by Temme [37], the asymptotics of this type of functions are quite difficult and hard to investigate. Nevertheless, it is possible to take advantage of the definition of the variance parameter by considering the asymptotic pdf of the Hermitian product phase difference (15). As detailed in Appendix E, by considering an infinite number of looks and $|\rho| \neq 0$, it can be demonstrated that 
In the same appendix, it is shown that, as $|\rho| \rightarrow 0$, the following first-order approximation for $\sigma_{\nu_{1}^{\prime}}^{2}$ and $\sigma_{\nu_{2}^{\prime}}^{2}$ can be employed:

$$
\sigma_{\nu_{1}^{\prime}}^{2}=\sigma_{\nu_{2}^{\prime}}^{2}=\frac{1}{2}\left(1-|\rho|^{2}\right)^{n}+O\left(|\rho|^{2}\right), \quad|\rho| \rightarrow 0
$$

Despite (27) results from a first-order approximation of the variances $\sigma_{\nu_{1}^{\prime}}^{2}$ and $\sigma_{\nu_{2}^{\prime}}^{2}$ in the vicinity of $|\rho|=0$, its results are interesting in highlighting that this curve may also be extended to the complete coherence range. Fig. 2 shows that $\sigma_{\nu_{1}^{\prime}}^{2}$ and $\sigma_{\nu_{2}^{\prime}}^{2}$ present similar values for the whole range of coherences. Consequently, (24) and (25) can be approximated by the following family of curves:

$$
\sigma_{\nu_{1}^{\prime}}^{2}=\sigma_{\nu_{2}^{\prime}}^{2} \simeq \frac{1}{2}\left(1-|\rho|^{2}\right)^{\alpha n}
$$

where $\alpha$ represents a constant value. The accuracy of this approximation can be observed in Fig. 2, where the parameter $\alpha=0.685$ has been employed. As observed, (28) offers a good approximation of the actual values of the variances $\sigma_{\nu_{1}^{\prime}}^{2}$ and $\sigma_{\nu_{2}^{\prime}}^{2}$, allowing to identify, in a very simple way, the type of dependence of these variances on the coherence $|\rho|$ and on the number of looks $n$.

The complex phasor noise model given by (19) admits an additional simplification by defining two novel noise sources as follows [38]:

$$
\begin{aligned}
& \nu_{c}=\nu_{1}^{\prime} \cos \left(\phi_{x}\right)-\nu_{2}^{\prime} \sin \left(\phi_{x}\right) \\
& \nu_{s}=\nu_{1}^{\prime} \sin \left(\phi_{x}\right)+\nu_{2}^{\prime} \cos \left(\phi_{x}\right) .
\end{aligned}
$$

By construction, the two noise sources $\nu_{c}$ and $\nu_{s}$ present a mean value equal to zero. Additionally, since the original noise sources $\nu_{1}^{\prime}$ and $\nu_{2}^{\prime}$ are uncorrelated, the variances of $\nu_{c}$ and $\nu_{s}$ result from the weighted average of the variances $\sigma_{\nu_{1}^{\prime}}^{2}$ and $\sigma_{\nu_{2}^{\prime}}^{2}$, as given by (29) and (30), according to the average phase $\phi_{x}$. Nevertheless, this dependence on $\phi_{x}$ can be neglected if the actual values of $\sigma_{\nu_{c}}^{2}$ and $\sigma_{\nu_{s}}^{2}$ are approximated by any value lying among $\sigma_{\nu_{1}^{\prime}}^{2}$ and $\sigma_{\nu_{2}^{\prime}}^{2}$. The best compromise for this approximation is to consider $\sigma_{\nu_{c}}^{2}$ and $\sigma_{\nu_{s}}^{2}$ to follow the family of curves of (28). Given this simplification, the noise model in (19) may be considered as a complex additive noise model

$$
\exp (j \phi)=N_{c} \exp \left(j \phi_{x}\right)+\left(\nu_{c}^{\prime}+j \nu_{s}^{\prime}\right)
$$

where all the useful information is contained in the first term of (31).

\section{B. Multilook Speckle Noise Model}

The previous analysis of the speckle noise model for the complex phasor $\exp (j \phi)$ is of importance since it allows obtaining the final speckle noise model for multilook multidimensional SAR data. As a preliminary conclusion, it may be stated that the behavior of the speckle noise, understood at this point as the variance characterizing the data, is determined essentially by the statistics associated to the phase. The speckle noise model for the Hermitian product of a pair of SAR images may be obtained by introducing (17) and (18) into (13)

$$
\begin{aligned}
& \Re\{z \exp (j \phi)\}=z N_{c} \cos \left(\phi_{x}\right)+z \nu_{1}^{\prime} \cos \left(\phi_{x}\right)-z \nu_{2}^{\prime} \sin \left(\phi_{x}\right) \\
& \Im\{z \exp (j \phi)\}=z N_{c} \sin \left(\phi_{x}\right)+z \nu_{1}^{\prime} \sin \left(\phi_{x}\right)+z \nu_{2}^{\prime} \cos \left(\phi_{x}\right) .
\end{aligned}
$$

By analyzing each additive term of (32) and (33), it is possible to characterize the speckle noise in the case of the Hermitian product. In parallel, it is also interesting to observe how the introduction of the Hermitian product amplitude $z$ changes the statistical and asymptotic behaviors of the different terms of (17) and (18), compared to (32) and (33).

The statistical behavior of the first additive term of (32) and (33) is determined by the statistics associated with the Hermitian product amplitude $z$. As a result, the first- and second-order moments of the first additive terms of (32) are

$$
\begin{aligned}
E\left\{z N_{c} \cos \left(\phi_{x}\right)\right\}= & N_{c} \cos \left(\phi_{x}\right) E\{z\} \\
= & \psi N_{c} \cos \left(\phi_{x}\right) \frac{\Gamma\left(\frac{3}{2}\right) \Gamma\left(n+\frac{1}{2}\right)}{n \Gamma(n)} 2 \\
& \times F_{1}\left(-\frac{1}{2}, \frac{1}{2}-n ; 1 ;|\rho|^{2}\right) \\
E\left\{\left(z N_{c} \cos \left(\phi_{x}\right)\right)^{2}\right\}= & N_{c}^{2} \cos ^{2}\left(\phi_{x}\right) E\left\{z^{2}\right\} \\
= & \psi^{2} N_{c}^{2} \cos ^{2}\left(\phi_{x}\right)\left(|\rho|^{2}+\frac{1}{n}\right)
\end{aligned}
$$

from where the variance can be derived. The first- and secondorder moments of the first additive term of (33) are just derived by interchanging $\cos \left(\phi_{x}\right)$ with $\sin \left(\phi_{x}\right)$ in the previous two equations. The asymptotic behavior for a large $n$ of the secondorder moment does not need a further analysis of (35). This is not the case for (34), where it is necessary to take into account, on the one hand, the asymptotics of the parameter $N_{c}$, and on the other hand, the asymptotics of the Hermitian product amplitude $z$. For the former parameter, (21)-(23) apply, whereas for the later, Tough et al. [22], based on [39], derived the following asymptotic expression for a large $n$ :

$$
E\{z\}=\psi\left(|\rho|+\frac{1}{n} \frac{\left(1-|\rho|^{2}\right)}{4|\rho|}\right) .
$$

The previous expression is useful, in combination with (35), in deriving a simple expression for the variance resulting from (34) and (35). If the asymptotic behavior of $N_{c}$ is not considered and only the terms of order $1 / n$ are retained in (36), it is possible to write

$$
\operatorname{var}\left\{z N_{c} \cos \left(\phi_{x}\right)\right\}=\psi^{2} N_{c}^{2} \cos ^{2}\left(\phi_{x}\right) \frac{\left(1+|\rho|^{2}\right)}{2 n} .
$$

Fig. 3 shows the actual value of the variance derived from (34) and (35) and the approximation provided by (37), which is characterized by a high accuracy. Finally, by means of (22) and (36), the following limit applies:

$$
\lim _{n \rightarrow \infty} E\left\{z N_{c} \cos \left(\phi_{x}\right)\right\}=\psi|\rho| \cos \left(\phi_{x}\right) .
$$




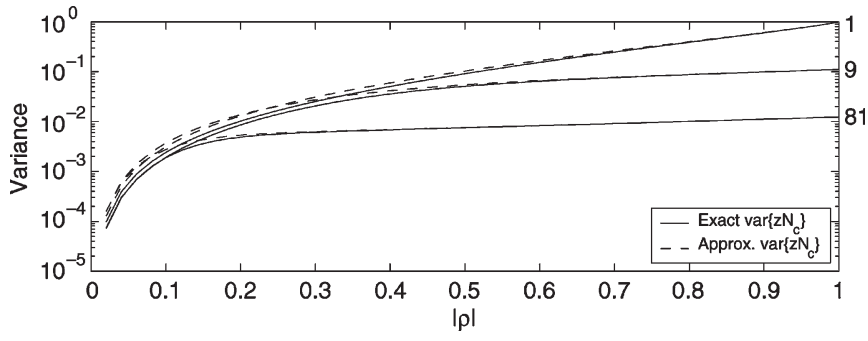

Fig. 3. $\operatorname{var}\left\{z N_{c} \cos \left(\phi_{x}\right)\right\}$ and approximated value for different number of looks ( $y$ axis is in log scale). A normalization $\psi=1, \phi_{x}=0$ is considered.

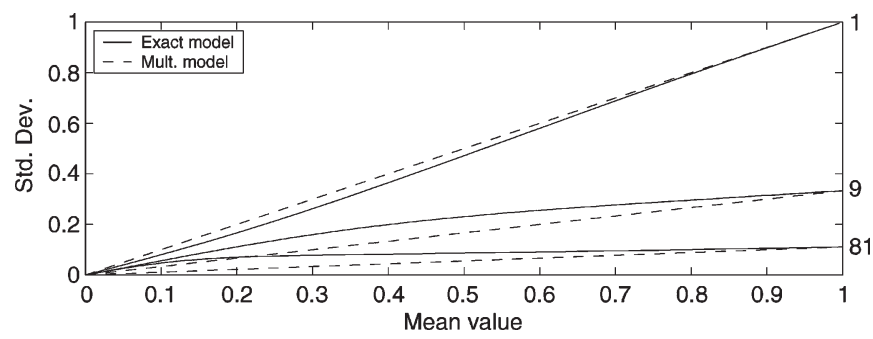

Fig. 4. Standard deviation versus mean values of the noise term $n_{m}$ for different number of looks $n$. A fully multiplicative model (dashed line) is included for comparison. A normalization $\psi=1, \phi_{x}=0$ is considered.

By means of (37)

$$
\lim _{\substack{n \rightarrow \infty \\|\rho|=0}} \operatorname{var}\left\{z N_{c} \cos \left(\phi_{x}\right)\right\}=0 .
$$

The analysis of the relation between the mean and standard deviation values permits the identification of possible noise sources in the data. This analysis allowed identifying, in the case of single-look data, a multiplicative noise source in the first additive terms of (32) and (33) [9]. The introduction of this noise term was possible owing to the effects due to the parameter $N_{c}$, since the relation between the mean and standard deviation values, in the case of the Hermitian product amplitude $z$, does not correspond to a multiplicative noise mechanism. The relation between the mean and standard deviation obtained from (34) and (35) is depicted, for different values of the number of looks $n$, in Fig. 4. In order to help the interpretability of the different lines, the relation corresponding to a fully multiplicative noise mechanism, averaged by $n$ uncorrelated samples and corresponding to a line with a slope equal to $1 / \sqrt{n}$, is also included as a dashed line. The first conclusion, which must be extracted from Fig. 4, is that the multiplicative noise component identified for single-look data cannot be considered for multilook data. A fully multiplicative component would underestimate data variance, especially for low and medium coherences. By paying attention to (34), (35), and (37), one may observe that the term $\psi \exp \left(j \phi_{x}\right)$ is multiplicative with respect to all the other components. This behavior allows writing

$$
z N_{c} \exp \left(j \phi_{x}\right)=\psi \exp \left(j \phi_{x}\right) n_{m}
$$

where $n_{m}$ represents a multiplicative noise component which must be understood as a generalization of the multiplicative noise component in the case of single-look data [9]. As observed in (40), the multiplicative nature of $n_{m}$ with respect to $\psi \exp \left(j \phi_{x}\right)$ does not depend on any signal parameter. In [9], this multiplicative nature was also extended to the expected

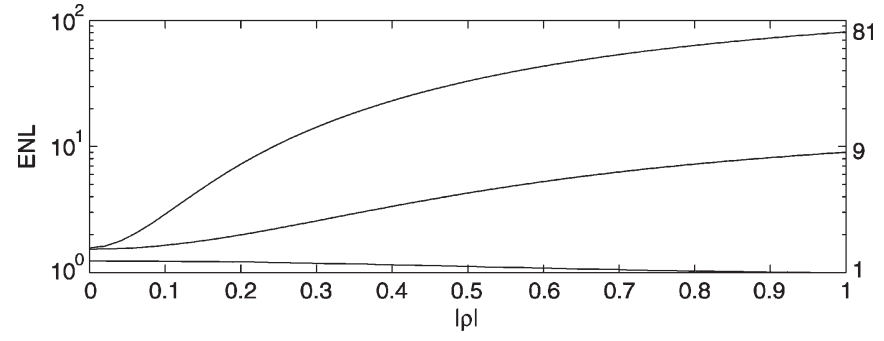

Fig. 5. Relation $E^{2}\left\{n_{m}\right\} / \operatorname{var}\left\{n_{m}\right\}$ of the noise term $n_{m}$ for different number of looks $n$ ( $y$ axis is in log scale).

value of the power normalized amplitude, denoted as $\bar{z}_{n}$. This later extension is no longer possible in the multilook case, so it is necessary to embed $N_{c} \bar{z}_{n}$ in $n_{m}$ as its mean value. As a result, $n_{m}$ may be characterized as follows:

$$
\begin{aligned}
E\left\{n_{m}\right\} & =N_{c} \bar{z}_{n} \\
\operatorname{var}\left\{n_{m}\right\} & =N_{c}^{2} \frac{\left(1+|\rho|^{2}\right)}{2 n}
\end{aligned}
$$

where $\bar{z}_{n}=E\{z\} / \psi$ and the approximation introduced in (37) has been considered to derive (42), which could have also been derived considering the actual value. Nevertheless, the high accuracy of this approximation allows a simple expression for the variance of $n_{m}$. In order to study the nature of the noise component $n_{m}$, it is possible to extend the idea of equivalent number of looks (ENL) employed to characterize the SAR image intensity. By considering (41) and (42), the quotient between $E^{2}\left\{n_{m}\right\}$ and $\operatorname{var}\left\{n_{m}\right\}$ is

$$
\frac{E^{2}\left\{n_{m}\right\}}{\operatorname{var}\left\{n_{m}\right\}}=2 n \frac{\bar{z}_{n}^{2}}{\left(1+|\rho|^{2}\right)}
$$

which, for a large $n$, reduces to

$$
\frac{E^{2}\left\{n_{m}\right\}}{\operatorname{var}\left\{n_{m}\right\}}=2 n \frac{|\rho|^{2}}{\left(1+|\rho|^{2}\right)} .
$$

Fig. 5 represents (43) for several values of $n$. For single-look data, (43) is almost constant in the whole coherence range, supporting the conclusions extracted by the study in [9]. However, as $n$ increases, (43) depends on $|\rho|$, in such a way that the lower the coherence, the lower is (43). For $|\rho|=1$, which corresponds to completely correlated SAR images, it is observed that (43) is equal to $n$, but it is also interesting to note that $E\left\{n_{m}\right\}=1$ and $\operatorname{var}\left\{n_{m}\right\}=1 / n$. Therefore, one can conclude that the first additive terms of (32) and (33) reduce to the multiplicative noise model for the SAR images intensity.

The second additive term of (32) contains the term $\nu_{1}^{\prime}=$ $\cos (\nu)-N_{c}$. On the basis of the analysis presented in the previous paragraph, one can show

$$
\begin{aligned}
E\left\{z \nu_{1}^{\prime} \cos \left(\phi_{x}\right)\right\}= & E\left\{\left(z \cos (\nu)-N_{c} E\{z\}\right)\right\} \cos \left(\phi_{x}\right) \\
= & \psi \cos \left(\phi_{x}\right) \\
& \times\left(|\rho|-N_{c} \frac{\Gamma\left(\frac{3}{2}\right) \Gamma\left(n+\frac{1}{2}\right)}{n \Gamma(n)}\right. \\
& \left.\quad \times{ }_{2} F_{1}\left(-\frac{1}{2}, \frac{1}{2}-n ; 1 ;|\rho|^{2}\right)\right) .
\end{aligned}
$$




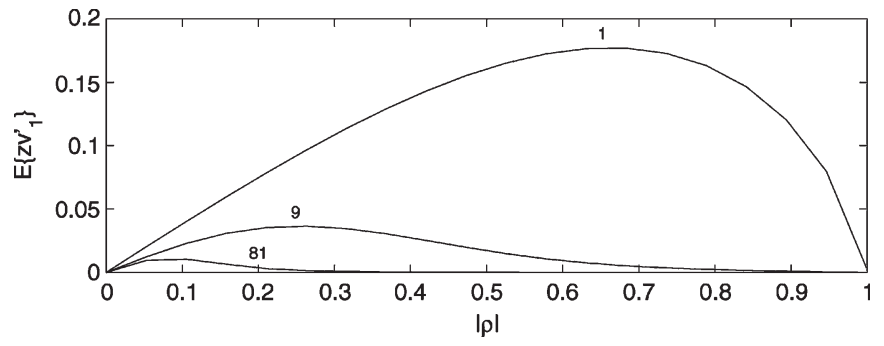

Fig. 6. Mean value $E\left\{z \nu_{1}^{\prime}\right\}$ for different number of looks $n$. A normalization $\psi=1, \phi_{x}=0$ is considered.

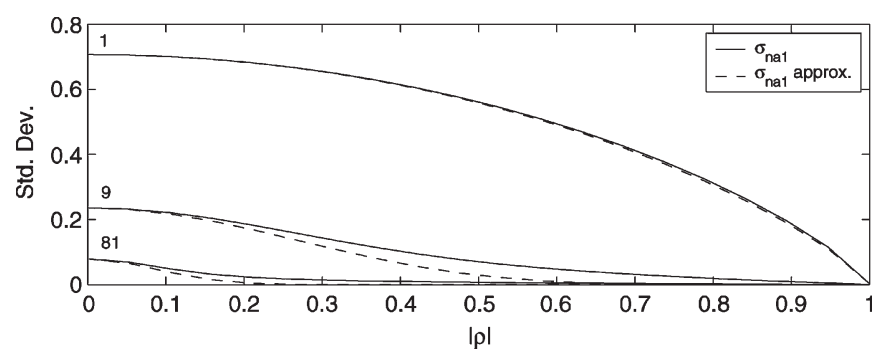

Fig. 7. Exact and approximated values of the standard deviation Std.Dev. $\left\{z \nu_{1}^{\prime} \cos \left(\phi_{x}\right)\right\}$ for different number of looks $n$. A normalization $\psi=1, \phi_{x}=0$ is considered.

Again, the expectation of the second additive term of (33) is obtained by interchanging $\cos \left(\phi_{x}\right)$ with $\sin \left(\phi_{x}\right)$ in the previous equation. Fig. 6 depicts the behavior of (45) for different values of $n$. Equation (45) presents such small values that they can be neglected for medium and high coherences for $n>16$. The asymptotics of (45) can be easily found by considering (36), giving rise to

$$
\lim _{n \rightarrow \infty} E\left\{z \nu_{1}^{\prime} \cos \left(\phi_{x}\right)\right\}=0
$$

The variance takes the following expression (47), shown at the bottom of the page.

The previous variance expression results, as observed, are extremely complicated. Thus, a simplification is of interest in order to determine the effects of the different parameters, which can be observed in detail in Figs. 7 and 8. Equation (47) is approximated by the same family of curves for the variance of the third additive term of (32) and (33)

$$
\operatorname{var}\left\{z \nu_{1}^{\prime} \cos \left(\phi_{x}\right)\right\}=\psi^{2} \cos ^{2}\left(\phi_{x}\right) \frac{1}{2 n}\left(1-|\rho|^{2}\right)^{1.64 n}
$$

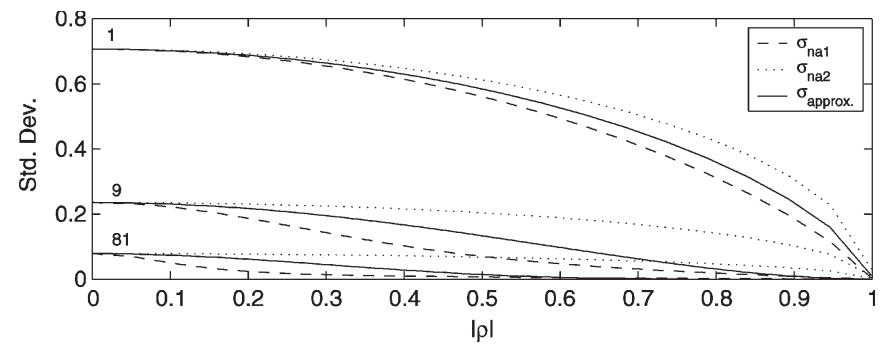

Fig. 8. Exact and approximated values of the standard deviation of the noise terms for different number of looks $n$. A normalization $\psi=1, \phi_{x}=0$ is considered.

in which the accuracy with respect to the real variance value can also be observed in Fig. 7. In the light of (48), it is easy to demonstrate the following limit:

$$
\lim _{n \rightarrow \infty} \operatorname{var}\left\{z \nu_{1}^{\prime} \cos \left(\phi_{x}\right)\right\}=0 .
$$

The limits in (46) and (49) demonstrate that the second additive term of (32) and (33) can be neglected for a large enough $n$.

Finally, by considering (45) and (48), the statistical behavior of the second additive term of (32) and (33) can be embedded in an additive zero-mean noise component $n_{a 1}$

$$
z \nu_{1}^{\prime} \exp \left(j \phi_{x}\right)=\psi\left\{\left(|\rho|-N_{c} \bar{z}_{n}\right)+n_{a 1}\right\} \exp \left(j \phi_{x}\right)
$$

such that

$$
\begin{aligned}
E\left\{n_{a 1}\right\} & =0 \\
\sigma_{n_{a 1}}^{2} & =\frac{1}{2 n}\left(1-|\rho|^{2}\right)^{1.64 n} .
\end{aligned}
$$

The analysis of the third additive terms of (32) results less complex than the other two terms, since they present a null expected value. This last term depends on the component $\nu_{2}^{\prime}=\sin (\nu)$. On the basis of the results presented in [22], it can be readily demonstrated that

$$
E\left\{z \nu_{2}^{\prime} \sin \left(\phi_{x}\right)\right\}=\sin \left(\phi_{x}\right) E\{z \sin (\nu)\}=0
$$

whereas the variance equals

$$
\begin{aligned}
\operatorname{var}\left\{z \nu_{2}^{\prime} \sin \left(\phi_{x}\right)\right\} & =\sin ^{2}\left(\phi_{x}\right) E\left\{\left(z \nu_{2}^{\prime}-E\left\{z \nu_{2}^{\prime}\right\}\right)^{2}\right\} \\
& =\psi^{2} \sin ^{2}\left(\phi_{x}\right) \frac{1}{2 n}\left(1-|\rho|^{2}\right) .
\end{aligned}
$$

$$
\begin{aligned}
\operatorname{var}\left\{z \nu_{1}^{\prime} \cos \left(\phi_{x}\right)\right\}= & E\left\{\left(z \nu_{1}^{\prime}-E\left\{z \nu_{1}^{\prime}\right\}\right)^{2}\right\} \cos ^{2}\left(\phi_{x}\right) \\
= & \left(E\left\{z^{2}\left(\cos (v)-N_{c}\right)^{2}\right\}-E^{2}\left\{z v_{1}^{\prime}\right\}\right) \cos ^{2}\left(\phi_{x}\right) \\
= & \psi^{2} \cos ^{2}\left(\phi_{x}\right)\left(\frac{\left(1-|\rho|^{2}\right)}{2 n}+\frac{n+1}{n}|\rho|^{2}+\frac{N_{c}^{2}}{n}\left(1+n|\rho|^{2}\right)-2 N_{c} \frac{\Gamma\left(\frac{5}{2}\right) \Gamma\left(\frac{2 n+3}{2}\right)}{n^{4} \Gamma(n)}|\rho|_{2} F_{1}\right. \\
& \left.\quad \times\left(\frac{1}{2}, \frac{1}{2}-n ; 2 ;|\rho|^{2}\right)-\left(|\rho|-N_{c} \frac{\Gamma\left(\frac{3}{2}\right) \Gamma\left(n+\frac{1}{2}\right)}{n \Gamma(n)} F_{2}\left(-\frac{1}{2}, \frac{1}{2}-n ; 1 ;|\rho|^{2}\right)\right)^{2}\right)
\end{aligned}
$$


The equivalent results in case of (33) are obtained by interchanging $\sin \left(\phi_{x}\right)$ with $\cos \left(\phi_{x}\right)$ in the previous two equations. As a result, the third additive term of (32) and (33) can be modeled by a zero-mean additive noise component

$$
z \nu_{2}^{\prime} \exp \left(j \phi_{x}\right)=\psi n_{a 2} \exp \left(j \phi_{x}\right)
$$

such that

$$
\begin{aligned}
E\left\{n_{a 2}\right\} & =0 \\
\sigma_{n_{a 2}}^{2} & =\frac{1}{2 n}\left(1-|\rho|^{2}\right) .
\end{aligned}
$$

One can observe that the variance characterizing the additive component $n_{a 2}$ is almost equal to the one corresponding to $n_{a 1}$. Fig. 8 presents also the behavior of the variance of $n_{a 2}$, where one can notice the similarity of the variance of the two additive noise mechanisms.

By considering (40), (50), and (55), the complex Hermitian product of two SAR images, (32) and (33), may be modeled as follows:

$$
\begin{array}{r}
\left\langle S_{i} S_{j}^{*}\right\rangle_{n}=\psi\left(n_{m}+\left(|\rho|-N_{c} \bar{z}_{n}\right)+\left(n_{a 1}+j n_{a 2}\right)\right) \exp \left(j \phi_{x}\right), \\
i, j=1,2, \ldots, m .
\end{array}
$$

This expression can be considered as exact in terms of variance, as no simplifications have been included in the model. Nevertheless, since $E\left\{n_{a 1}\right\}=0$ and $E\left\{n_{a 2}\right\}=0$, only the two first additive terms of (58) provide useful information. This fact suggest a simplification based on eliminating the dependence of the complex term $n_{a 1}+j n_{a 2}$ on the phase term $\exp \left(j \phi_{x}\right)$.

The Hermitian product $\left\langle S_{i} S_{j}^{*}\right\rangle_{n}$ presents two independent parameters, i.e., its real and imaginary parts or its amplitude and phase, depending on the selected parameterization. Nonetheless, (58) contains three random terms, which consequently are not uncorrelated. Thus, it is necessary to analyze the covariance between these three additive terms. The covariance of the first and second additive terms of (32) and (33) can be expressed as follows:

$$
\begin{aligned}
& E\left\{\left(z N_{c} \cos \left(\phi_{x}\right)-\right.\right.\left.E\left\{z N_{c} \cos \left(\phi_{x}\right)\right\}\right) \\
& \times\left(z \nu_{1}^{\prime} \cos \left(\phi_{x}\right)-\right.\left.\left.E\left\{z \nu_{1}^{\prime} \cos \left(\phi_{x}\right)\right\}\right)\right\} \\
&=\cos ^{2}\left(\phi_{x}\right)\left(N_{c} E\left\{z^{2} \cos (\nu)\right\}-N_{c} E\{z\}\right. \\
&\left.\times E\left\{z\left(\cos (\nu)-N_{c}\right)\right\}\right)
\end{aligned}
$$

which is an expression that may be derived from the previous results. Fig. 9 details this covariance for several values on $n$. From this figure, one can observe that this covariance term presents small values and, additionally, it can be neglected for analysis purposes for $n>9$. The other two covariance terms relate the first and the third additive terms

$$
E\left\{\cos \left(\phi_{x}\right)\left(z N_{c}-E\left\{z N_{c}\right\}\right) \sin \left(\phi_{x}\right)\left(z \nu_{2}^{\prime}\right)\right\}=0
$$

and the second and third additive terms

$$
E\left\{\cos \left(\phi_{x}\right)\left(z \nu_{1}^{\prime}-E\left\{z \nu_{1}^{\prime}\right\}\right) \sin \left(\phi_{x}\right)\left(z \nu_{2}^{\prime}\right)\right\}=0 .
$$

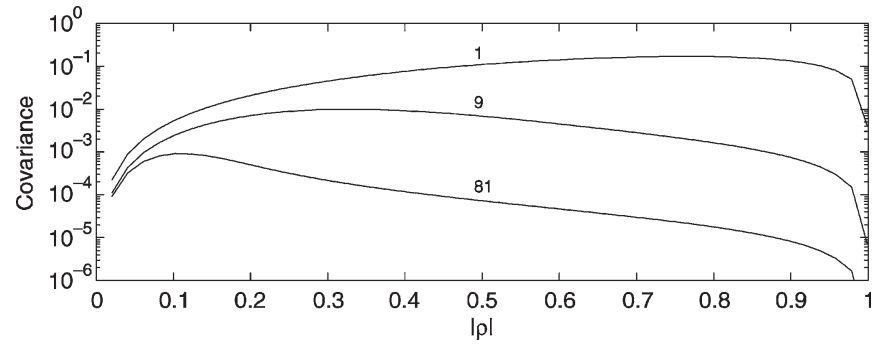

Fig. 9. Covariance term $E\left\{\left(z N_{c} \cos \left(\phi_{x}\right)-E\left\{z N_{c} \cos \left(\phi_{x}\right)\right\}\right)\right.$ $\left.\left(z \nu_{1}^{\prime} \cos \left(\phi_{x}\right)-E\left\{z \nu_{1}^{\prime} \cos \left(\phi_{x}\right)\right\}\right)\right\}$ for different number of looks $n(y$ axis is in $\log$ scale). A normalization $\psi=1, \phi_{x}=0$ is considered.

\section{Multilook Speckle Noise Model Simplification}

The simplification of the multilook speckle noise model follows the simplification of the single-look speckle noise model. As it can be observed, the complex term $\exp \left(j \phi_{x}\right)$ produces a combination of the real and imaginary parts of the complex additive noise component $n_{a 1}+j n_{a 2}$

$$
\begin{aligned}
& n_{\mathrm{ar}}=n_{a 1} \cos \left(\phi_{x}\right)-n_{a 2} \sin \left(\phi_{x}\right) \\
& n_{\mathrm{ai}}=n_{a 1} \sin \left(\phi_{x}\right)+n_{a 2} \cos \left(\phi_{x}\right) .
\end{aligned}
$$

From the previous equations, one gets $E\left\{n_{\mathrm{ar}}\right\}=0$ and $E\left\{n_{\mathrm{ai}}\right\}=0$. In addition, the variance of the terms $n_{\mathrm{ar}}$ and $n_{\mathrm{ai}}$ presents a clear dependence on the phase $\phi_{x}$, but the variance of the terms $n_{a 1}$ and $n_{a 2}$ is close enough to neglect this dependence by considering the following approximation:

$$
\sigma_{n_{\mathrm{ar}}}^{2}=\sigma_{n_{\mathrm{ai}}}^{2}=\frac{1}{2 n}\left(1-|\rho|^{2}\right)^{1.32 \sqrt{n}} .
$$

The previous curve can be considered as an average value of the variances of $n_{a 1}$ and $n_{a 2}$, as detailed in Fig. 8 (solid line). Hence, by introducing (62) and (63) into (58), one gets the following multilook speckle noise model for the Hermitian product of two SAR images:

$$
\begin{array}{r}
\left\langle S_{i} S_{j}^{*}\right\rangle_{n}=\psi n_{m} \exp \left(j \phi_{x}\right)+\psi\left(|\rho|-N_{c} \bar{z}_{n}\right) \exp \left(j \phi_{x}\right) \\
+\psi\left(n_{\mathrm{ar}}+j n_{\mathrm{ai}}\right), \quad i, j=1,2, \ldots, m .
\end{array}
$$

The previous model has been obtained based on the consideration that $n$ is the number of averaged samples. In case of real SAR data, $n$ should be substituted by the ENL of the filtered data. As done for the single-look case, (65) can be rewritten as follows:

$$
\begin{array}{r}
\left\langle S_{i} S_{j}^{*}\right\rangle_{n}=\psi|\rho| \exp \left(j \phi_{x}\right)+\psi\left(n_{m}-N_{c} \bar{z}_{n}\right) \exp \left(j \phi_{x}\right) \\
+\psi\left(n_{\mathrm{ar}}+j n_{\mathrm{ai}}\right), \quad i, j=1,2, \ldots, m
\end{array}
$$

yielding to the multilook multidimensional speckle noise model for the sample covariance matrix

$$
\mathbf{Z}_{n}=\mathbf{C}+\mathbf{N}_{m}+\mathbf{N}_{a}
$$

where, again, the matrix $\mathbf{N}_{m}$ is constructed straightforwardly from the second additive term of (66) and $\mathbf{N}_{a}$ from the third one. Equation (67) represents the extension of the multidimensional model (11) to the multilook data case. 


\section{Analysis of the Multilook Multidimensional SPECKLE NOISE MODEL}

The detailed analysis of (65) follows, in a parallel way, the analysis of the single-look speckle noise model already presented in [9]. Nevertheless, in what it follows, the emphasis will be on the effects of the number of looks in the different terms of (65) and especially on the multiplicative noise term $n_{m}$. First, (65) is particularized to consider the diagonal elements of the sample covariance matrix in (4). In this case, it can be considered that both SAR images present a complex correlation coefficient equal to $1 \exp (j 0)$, which when introduced into (65) results in

$$
\left\langle S_{i} S_{j}^{*}\right\rangle_{n}=\psi n_{m}, \quad i=1,2, \ldots, m
$$

where

$$
\begin{aligned}
E\left\{n_{m}\right\} & =1 \\
\operatorname{var}\left\{n_{m}\right\} & =\frac{1}{n}
\end{aligned}
$$

on the basis of (20), (41), and (42). The previous equations correspond to the multiplicative speckle noise model for the SAR images intensity. Thus, (65) may be considered as an extension of the multilook multiplicative speckle noise model.

The effects of the multiplicative speckle noise component $n_{m}$ must be analyzed according to $|\rho|$, but also with respect to the multiplicative term $\psi \exp \left(j \phi_{x}\right)$. By considering the mean value of $n_{m}$, i.e., (41), but also the variance in (42), one can observe that the influence of this noise term is proportional to $|\rho|$, in such a way that the higher the coherence, the higher is the influence, disappearing for the particular value $|\rho|=0$. In the same way, one can observe that the higher the number of looks $n$, the lower is the variance of the term $n_{m}$. A second effect which needs to be carefully considered is the repercussion of the multiplicative term $\psi \exp \left(j \phi_{x}\right)$. For a fixed value of $|\rho|$, (43) [or the asymptotic simplification in (44)] determines the slope of the curve relating the mean and variance. Therefore, the lower the coherence, the lower is the ratio $E^{2}\left\{n_{m}\right\} / \operatorname{var}\left\{n_{m}\right\}$. This fact means that this slope will increase for decreasing coherences. As detailed in the previous section, a fully multiplicative noise mechanism would not be able to take into account all the variance of the first additive terms of (32) and (33). Finally, the modulation by the complex phase term $\exp \left(j \phi_{x}\right)$ is analyzed. The real and imaginary parts of the first additive terms of (32) and (33) are modulated by $\cos \left(\phi_{x}\right)$ and $\sin \left(\phi_{x}\right)$, respectively. Since the multiplicative noise term $n_{m}$ affects only the amplitude of the Hermitian product, this dependence means that the noise source $n_{m}$ is also modulated by them. Thus, depending on the value of the phase $\phi_{x}$, it also determines the influence of the multiplicative speckle term in the final Hermitian product. This dependence on $\phi_{x}$ has important consequences, especially in interferometric applications. In such a case, it is possible to conclude that the topographic information can determine the nature and effects of speckle noise in the Hermitian product of the two SAR images.

The second term to analyze in (65) is the complex additive term $\psi\left(n_{a 1}+j n_{a 2}\right)$. Since the terms $n_{\text {ar }}$ and $n_{\text {ai }}$ present a mean equal to zero, the sole effect is introduced through a variance term. By considering the variance expressions in

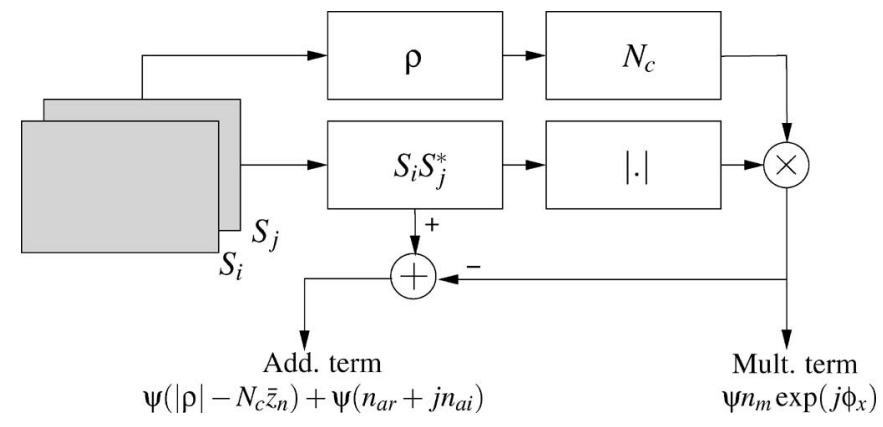

Fig. 10. Data processing scheme to separate the multiplicative (Mult.) and additive (Add.) terms of the Hermitian product of two complex SAR images $S_{i} S_{j}^{*}$.

(52) and (57), one can deduce that the higher the coherence, the lower is the influence of these additive noise terms. It is also worth to notice that this dependence is contrary to the dependence of the term $n_{m}$ on $|\rho|$. The term $\psi\left(n_{a 1}+j n_{a 2}\right)$ disappears completely for $|\rho|=1$, as the mean and variance values are equal to zero. Finally, this complex additive term is not influenced by the phase $\phi_{x}$ if one considers the noise model given at (65). Nevertheless, as previously demonstrated, if one considers the actual variance values, a small dependence $\phi_{x}$ can be observed.

Considering all the previous study, it is possible to conclude that the final nature of the speckle noise, in the case of the Hermitian product of two SAR images, is determined by the complex quantity $\rho$. In a simple way, it can be affirmed that for low coherences, the variance of the real and imaginary parts of the Hermitian product of two SAR images can be explained by the complex additive speckle noise term $\psi\left(n_{a 1}+j n_{a 2}\right)$. However, the variance at high coherences is basically due to the multiplicative term $n_{m}$. In this situation, it is also important to take into account the dependence on the phase $\phi_{x}$, as this phase modulates the influence of $n_{m}$, in such a way that, in some cases, depending on $\phi_{x}$, the data variance for high coherence is due to the additive term $\psi\left(n_{a 1}+j n_{a 2}\right)$.

\section{EXPERIMENTAL Results}

The analysis of the model proposed in (58) or (65) makes it necessary to divide the Hermitian product into the different components of the model. In what it follows, (65) is considered. In this case, the first additive term, due to its dependence on the noise component $n_{m}$, will be referred as the multiplicative term of the noise model. Hence, the other two additive terms will be called, consequently, the additive term of the model. In Section III, it has been demonstrated that speckle noise, in the case of the Hermitian product of two SAR images, is governed by the complex correlation coefficient characterizing the product. Therefore, this parameter must be estimated to split the Hermitian product into the two previous terms. The way they are obtained is drawn in Fig. 10. On the basis of the estimated complex correlation coefficient $\rho$, the multiplicative term is obtained by multiplying the amplitude of the Hermitian product by $N_{c}$, whereas the additive term is generated by subtracting this multiplicative term from the Hermitian product. Based on the processing scheme depicted in Fig. 10, the multilook speckle noise model has been tested on an experimental small-baseline L-band PolSAR dataset. These data have been 


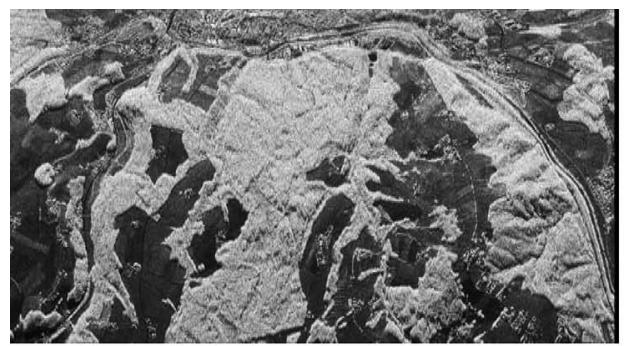

(a)

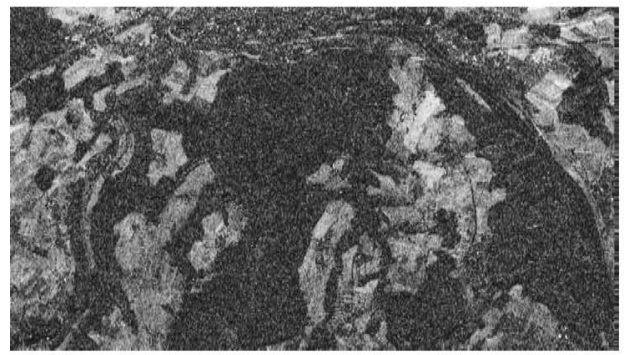

(c)

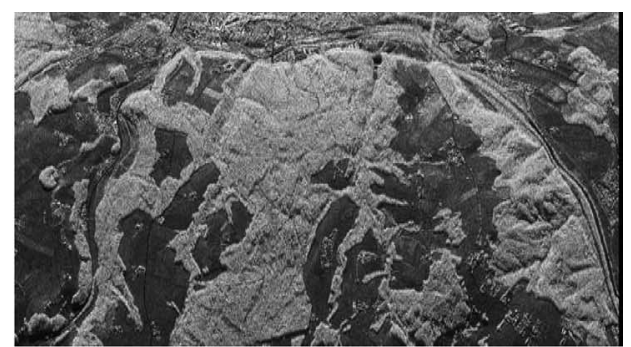

(b)

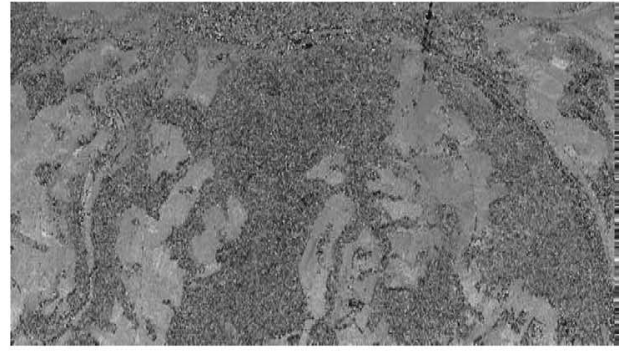

(d)

Fig. 11. ESAR Traunstein PolSAR dataset. Coherence parameters are obtained with a $7 \times 7$ square window. (a) $\left|S_{\text {hh }}\right|$. (b) $\left|S_{\nu \nu}\right|$. (c) $|\rho|$. (d) $\phi_{x}$.

acquired by the ESAR sensor, operated by German Aerospace Center (DLR), over the Traunstein area located in southern Germany. Fig. 11(a) and (b) presents the amplitudes of the $S_{\mathrm{hh}}$ and $S_{\nu \nu}$ channels, where agricultural and forested zones can be identified, and an urban area in located on the top of the image. Fig. 11(c) and (d) presents the complex correlation coefficient of $S_{\mathrm{hh}} S_{\nu \nu}^{*}$.

The dispersion diagram is an interesting tool allowing the analysis of noise mechanisms present in experimental data. This tool plots the standard deviation as a function of the data mean, both calculated in a given area considered as homogeneous and normally in the range of about ten pixels wide. The final dispersion diagram is obtained by plotting the pairs standard deviation versus the mean of the whole dataset, considering also their density.

Fig. 12 represents the different dispersion diagrams derived from the multilook speckle noise model (65), corresponding to the quantity $\Re\left\{\left\langle S_{\mathrm{hh}} S_{\nu \nu}^{*}\right\rangle_{81}\right\}$ of the Traunstein dataset, assuming a multilook of 81 pixels, obtained by $9 \times 9$ pixel window. The figure rows represent the dispersion diagrams for different coherence ranges, whereas columns correspond to the different terms of (65): the total dispersion diagram of $\Re\left\{\left\langle S_{\mathrm{hh}} S_{\nu \nu}^{*}\right\rangle_{81}\right\}$ and the dispersion diagrams of the corresponding multiplicative and additive terms (see Fig. 10). Additionally, the solid lines in Fig. 12 represent a multiplicative relation among the standard deviation and the mean, i.e., a line with a slope equal to $1 / \sqrt{n}$, where in this case $n=81$. Dashed lines in the first and second columns indicate a multiplicative relation, in which the slope is determined by the relation presented in (43).

In a general outline, it can be considered that low correlation data are affected by the additive speckle noise component, whereas for high-correlation data, speckle is characterized by a multiplicative noise mechanism. Nevertheless, this observation needs from an in-depth analysis. By paying attention to the multiplicative speckle term column, one can observe the variation of the multiplicative term slope as a function of coherence. For low coherences, the line slope does not agree with $1 / \sqrt{n}$ but with a lower one. The value of the exact slope matches with the one determined by (43) since the higher densities (represented in black) are on this line. Thus, one can conclude that data coherence determines the nature of the multiplicative speckle mechanism. It is important to highlight that this special mechanism, due to the small dependence on $|\rho|$ (see Fig. 5), has been revealed by the data averaging process. The additive speckle component, on the other hand, does not present any variation with respect to the single-look data case, except for the reduction in a value $1 / \sqrt{n}$ of the standard deviation value.

The previous analysis has shown the dependence of the filtered speckle noise on the coherence characterizing the Hermitian product of two SAR images. The next analysis, on the contrary, focuses on the study of the multilook speckle noise for data processed with two different values of the number of averaged samples $n$, given a fixed coherence value. This paper concentrates on an 8251-pixel homogeneous area of the Traunstein site, characterized by an average sample coherence of $|\rho|=0.57 \pm 0.11$ and an average phase of $\phi_{x}=1.09 \pm$ $0.28 \mathrm{rad}$. PolSAR data have been filtered by a $3 \times 3$ (nine-look) and a $9 \times 9$ (81-look) pixel windows. These results are depicted in Fig. 13, where the power normalized mean and standard deviation values of the multiplicative and additive speckle components of the quantities $\Re\left\{\left\langle S_{\mathrm{hh}} S_{\nu \nu}^{*}\right\rangle_{n}\right\}$ and $\Im\left\{\left\langle S_{\mathrm{hh}} S_{\nu \nu}^{*}\right\rangle_{n}\right\}$ are shown as a function of coherence. Solid lines represent the theoretical values of the different parameters as a function of coherence, whereas in the case of the standard deviation plots, dashed lines represent the approximation introduced in (64). The first conclusion that can be extracted after observing Fig. 13 is that averaged data fully agree with the theoretical values obtained in Section III. Comparing the different plots referring to the mean values of the multiplicative and additive speckle components, it can be observed that they do not vary with $n$, as expected. Nevertheless, the modulation induced by the phase $\phi_{x}$ is clearly noticeable, especially in the plots of the mean values of the multiplicative speckle noise term. In addition, as 

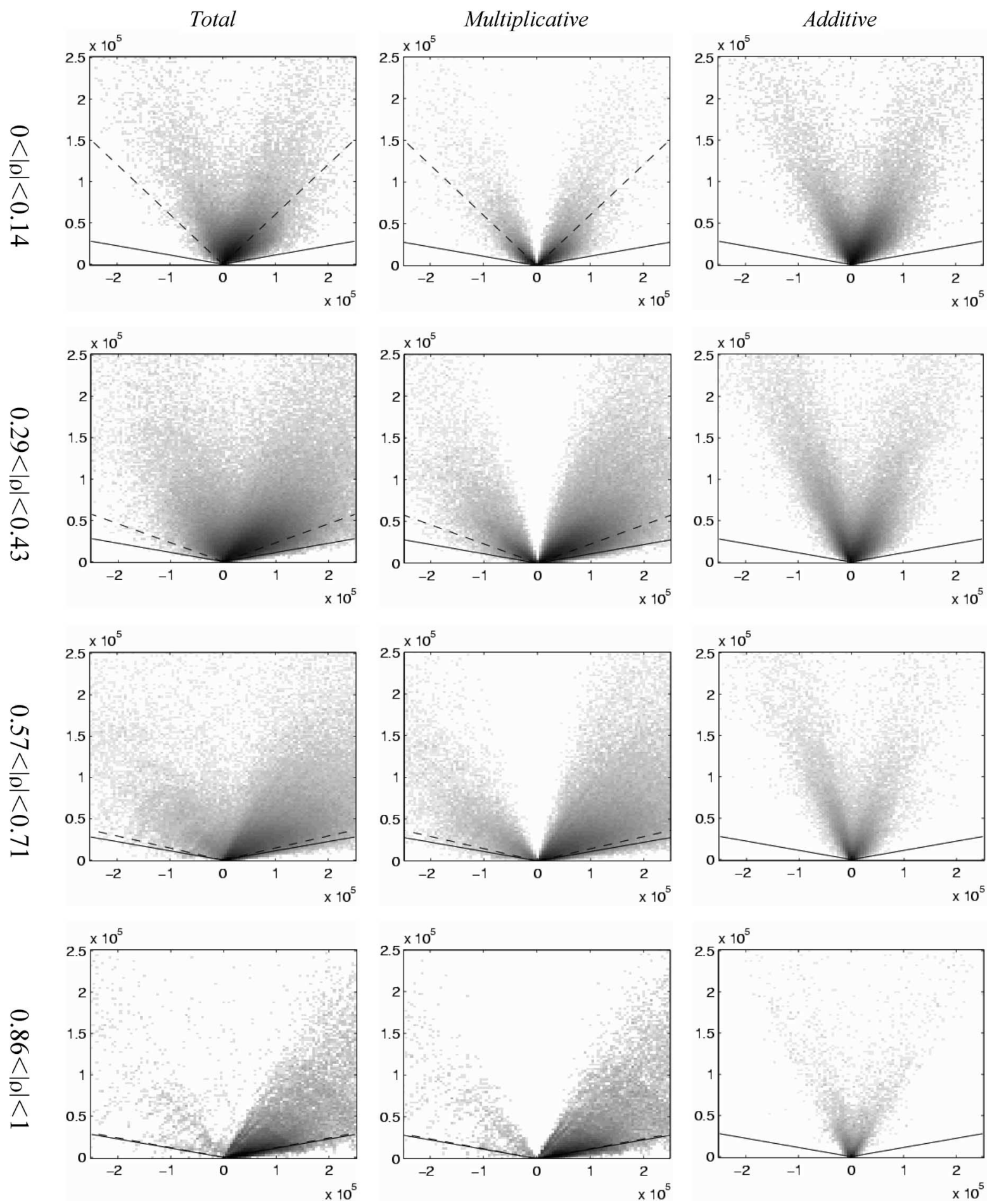

Fig. 12. Dispersion diagrams of the 81-look term $\Re\left\{\left\langle S_{\mathrm{hh}} S_{\nu \nu}^{*}\right\rangle_{81}\right\}$ corresponding to the Traunstein dataset. Dashed lines represents a line with a slope equal to $1 / 9$, whereas solid lines have a slope equal to $2 n \bar{z}_{n}^{2} /\left(1+|\rho|^{2}\right)$. The $x$ axis represents the mean value and the $y$ axis the standard deviation.

detailed theoretically in Fig. 6, the mean value of the additive terms can be neglected, even for nine-look data. The effect of the multilook process is, however, evident in the case of the standard deviation plots, where as expected, 81-look data present lower standard deviation values than nine-look data for both the multiplicative and additive speckle noise components. In the same way as the mean values, the standard deviation values of the multiplicative speckle component are affected by the modulation induced by the phase term $\phi_{x}$. This modulation is also visible in the case of the additive speckle term as the difference between the solid and dashed lines. This modulation of the phase term $\phi_{x}$ is especially important in interferometric applications, since in this case, it may contain topographic information. Therefore, the topography of the terrain may determine the final nature of the speckle noise as it acts on the combination of the multiplicative and additive speckle components. 

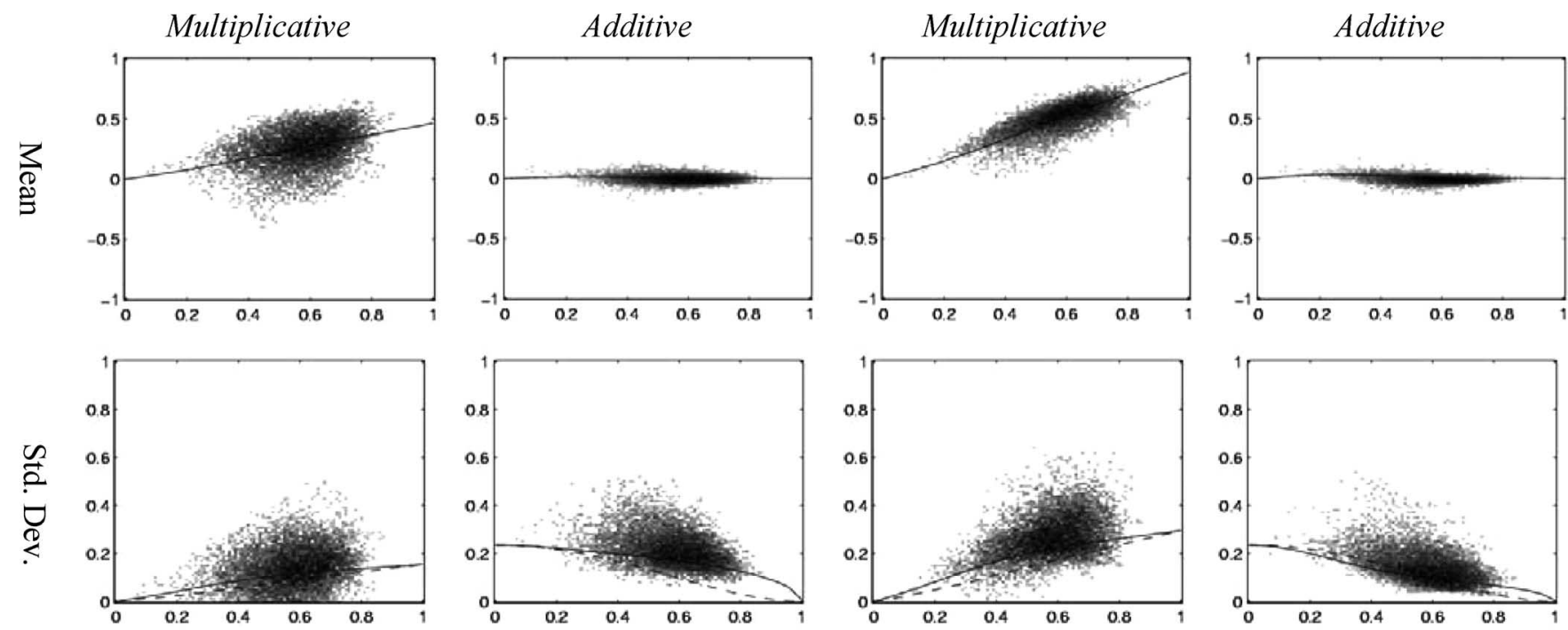

(a)

(b)
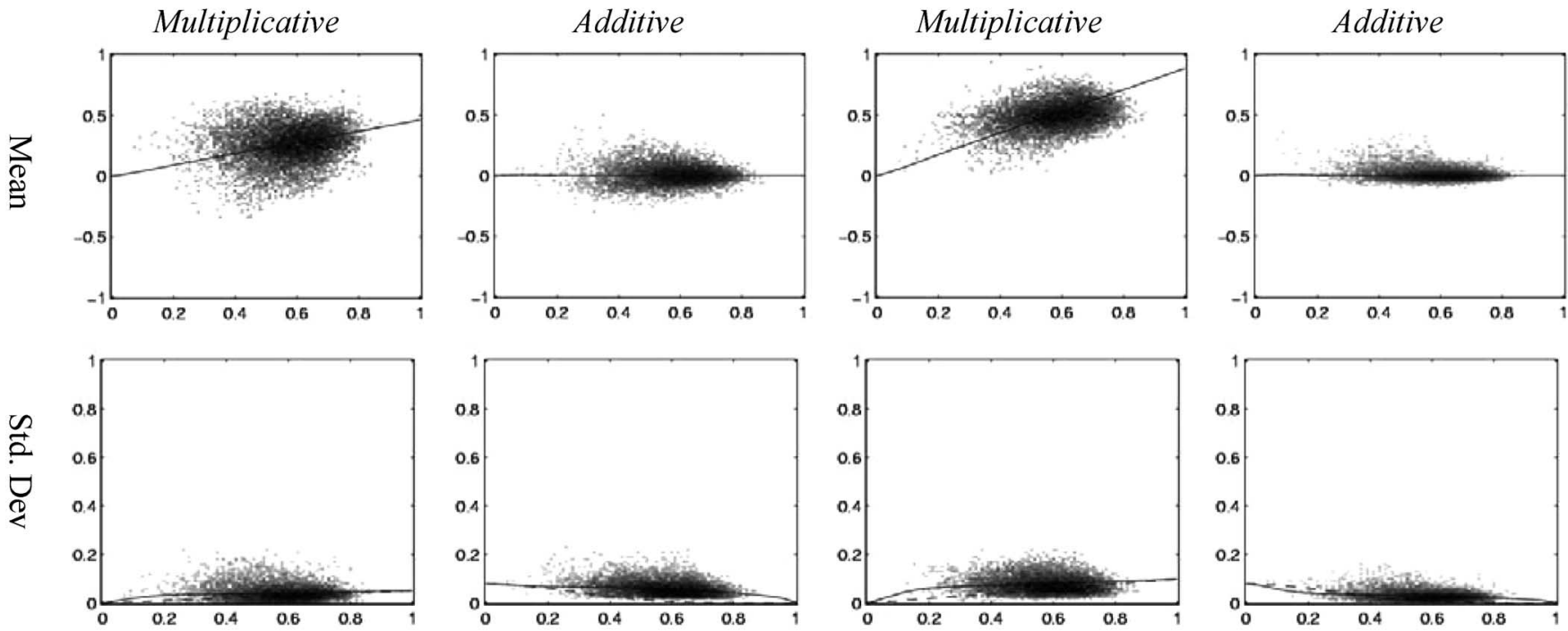

(c)
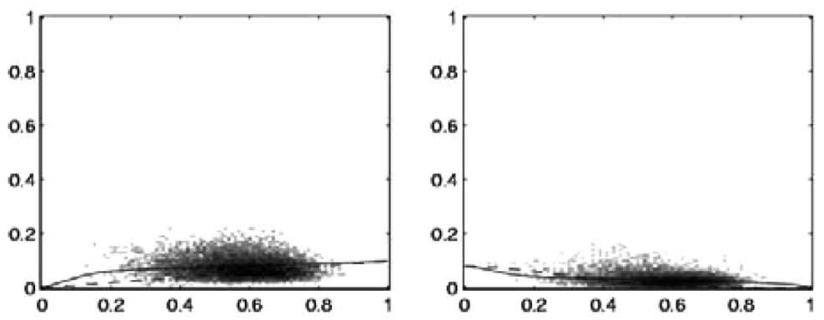

(d)

Fig. 13. Density plots for the mean and standard deviation of the different terms of the multilook speckle noise model for the Traunstein dataset in an area where the average coherence is equal to $\rho=0.57 \exp (j 1.09)$. The $x$ axis coordinates represent the coherence $|\rho|$. Quantities have been normalized by $\psi$. Solid lines represent theoretical values. Dashed lines correspond to approximations. (a) $\Re\left\{\left\langle S_{\mathrm{hh}} S_{\nu \nu}^{*}\right\rangle_{9}\right\}$. (b) $\Im\left\{\left\langle S_{\mathrm{hh}} S_{\nu \nu}^{*}\right\rangle_{9}\right\}$. (c) $\Re\left\{\left\langle S_{\mathrm{hh}} S_{\nu \nu}^{*}\right\rangle_{81}\right\}$. (d) $\left\{\left\langle\left\langle S_{\mathrm{hh}} S_{\nu \nu}^{*}\right\rangle_{81}\right\}\right.$.

Another important effect of this phase modulation is observed if one compares the plots of $\Re\left\{\left\langle S_{\mathrm{hh}} S_{\nu \nu}^{*}\right\rangle_{n}\right\}$ and $\Im\left\{\left\langle S_{\mathrm{hh}} S_{\nu \nu}^{*}\right\rangle_{n}\right\}$. By considering first the term $\Re\left\{\left\langle S_{\mathrm{hh}} S_{\nu \nu}^{*}\right\rangle_{n}\right\}$, the standard deviation values corresponding to the additive term are slightly higher than those of the multiplicative speckle term, allowing the conclusion that speckle shall be dominated by additive behavior. Nevertheless, this is not th e case for $\Im\left\{\left\langle S_{\mathrm{hh}} S_{\nu \nu}^{*}\right\rangle_{n}\right\}$, where the multiplicative speckle term presents larger values.

\section{CONCLUSION}

This paper presents an extension of the single-look multidimensional speckle noise model to the multilook data case. The extension itself has also been considered in the asymptotic case, with respect to the number of looks, demonstrating the coherence and validity of the proposed speckle noise model.
With respect to the single-look multidimensional speckle noise model case, the introduction of the averaging process has made necessary the inclusion of some variations on the original model. These variations affect essentially the multiplicative speckle noise source. The averaging process prevents, as demonstrated, to consider a fully multiplicative noise source, although this multiplicative nature is still present with respect to the parameter $\psi \exp (j \phi)$. Despite these changes, the philosophy of the original single-look multidimensional speckle noise model is still present in the multilook speckle noise model. In the multilook case and for the Hermitian product of two SAR images, the speckle noise is due to the combination of a real multiplicative and a complex additive speckle noise components. The combination of these components is determined by the complex correlation coefficient characterizing the Hermitian product. Speckle noise presents an 
additive nature for low coherences, whereas it is multiplicative for high ones. In the extreme case of coherence equal to one, the proposed model reduces to the classical multiplicative speckle noise model for the SAR images intensity. It is important to highlight the dependence of this combination on the phase information. This influence provokes differences among the real and imaginary parts of the speckle noise. The most interesting conclusion here concerns interferometric applications, since the final combination of the speckle shall depend on the terrain topography, as this topography determines the value of the phase information.

\section{APPENDIX A}

\section{Phase Difference Asymptotic Distribution}

The phase pdf in (14) contains two terms whose asymptotic behavior for a large $n$ is next analyzed. Without loss of generality, this development will consider $\phi_{x}=0$. The asymptotic behavior of the first term

$$
p_{\phi, 1}(\phi)=\frac{\Gamma(n+1 / 2)\left(1-|\rho|^{2}\right)^{n} \beta}{2 \sqrt{\pi} \Gamma(n)\left(1-\beta^{2}\right)^{n+1 / 2}}
$$

follows from the asymptotic behavior of the ratio of gamma functions. The ratio of the gamma functions in (A1) presents the following asymptotic series expansion [40]:

$\frac{\Gamma(J+1 / 2)}{\Gamma(J)}=\sqrt{J}\left(1-\frac{1}{8 J}+\frac{1}{128 J^{2}}+\frac{5}{1024 J^{3}}-\frac{21}{32768 J^{4}}+\ldots\right)$

in which the asymptotic behavior for a large $J$ is determined by the first term, since the rest of the terms are, at least, of the order $1 / J$. Introducing (A2) into (A1), for a large $n$

$$
p_{\phi, 1}(\phi)=\frac{\sqrt{n}\left(1-|\rho|^{2}\right)^{n} \beta}{2 \sqrt{\pi}\left(1-\beta^{2}\right)^{n+1 / 2}}
$$

The analysis of the second term

$$
p_{\phi, 2}(\phi)=\frac{\left(1-|\rho|^{2}\right)^{n}}{2 \pi}{ }_{2} F_{1}\left(n, 1 ; \frac{1}{2} ; \beta^{2}\right)
$$

is needed from the asymptotic analysis of the Gauss hypergeometric function ${ }_{2} F_{1}(a, b ; c ; z)$. Using the transformation formula of hypergeometric functions [34]

$$
\begin{aligned}
{ }_{2} F_{1}(a, b ; c ; z)= & e^{b \pi j} \frac{\Gamma(c) \Gamma(a-c+1)}{\Gamma(a+b-c+1) \Gamma(c-b)} z^{-b} \\
& \times{ }_{2} F_{1}\left(b, b-c+1 ; a+b-c+1 ; 1-\frac{1}{z}\right) \\
& +\frac{\Gamma(c) \Gamma(a-c+1)}{\Gamma(b) \Gamma(a-b+1)} z^{b-c}(1-z)^{c-a-b} \\
& \times{ }_{2} F_{1}\left(1-b, c-b ; a-b+1 ; \frac{1}{z}\right)
\end{aligned}
$$

one can write (A5) as follows:

$$
\begin{array}{r}
p_{\phi, 2}(\phi)=\frac{\left(1-|\rho|^{2}\right)^{n}}{2 \pi}\left\{\frac{1}{(2 n+1) \beta^{2}}{ }_{2} F_{1}\left(1, \frac{3}{2} ; n+\frac{3}{2} ; 1-\frac{1}{\beta^{2}}\right)\right. \\
\left.+\frac{\sqrt{\pi \beta^{2}}}{\left(1-\beta^{2}\right)^{n+1 / 2}} \frac{\Gamma(n+1 / 2)}{\Gamma(n)}\right\} .
\end{array}
$$

Now, it is necessary to determine the behavior of (A6) for a large $n$ when the other parameters, especially $\beta$, are considered fixed. When $\beta$ is not close to zero, i.e., $|\rho| \neq 0$ and $\phi \neq$ $m(\pi / 2)$ for $m \in \mathbb{Z}$, the hypergeometric function in (A6) can be expanded in the standard power series, whether or not it is convergent, to obtain the asymptotic expansion for a large $n$. Nevertheless, since this term is at least of the order $1 / n$, the general behavior is determined by the term $\left(1-\beta^{2}\right)^{-n-1 / 2}$. Thus, by introducing (A6) into (A4), for a large $n$

$$
p_{\phi, 2}(\phi)=\frac{\sqrt{n}\left(1-|\rho|^{2}\right)^{n} \sqrt{\beta^{2}}}{2 \sqrt{\pi}\left(1-\beta^{2}\right)^{n+1 / 2}}, \quad|\rho| \cos (\phi) \neq 0 .
$$

As a result, by considering the expressions of (A3) and (A7), the asymptotic behavior of the Hermitian product phase difference is obtained, as expressed in (15).

\section{APPENDIX B}

EXPRESSIONS OF $N_{c}$ AND $N_{s}$

By considering $\phi$ as described by the distribution in (14), $N_{c}$ is obtained as follows:

$$
N_{c}=\int_{-\pi}^{\pi} \cos (\phi) p_{\phi}(\phi) d \phi .
$$

By introducing (14) into (B1), the following two integrals result:

$$
\begin{aligned}
& \frac{1}{2 \pi}\left(1-|\rho|^{2}\right)^{n} \int_{-\pi}^{\pi} \cos (\phi)_{2} F_{1}\left(n, 1 ; \frac{1}{2} ;|\rho|^{2} \cos ^{2}\right) d \phi \\
& \frac{\Gamma(n+1 / 2)}{2 \sqrt{\pi} \Gamma(n)}\left(1-|\rho|^{2}\right)^{n}|\rho| \int_{-\pi}^{\pi} \frac{\cos ^{2}(\phi)}{\left(1-|\rho|^{2} \cos ^{2}\right)^{n+1 / 2}} d \phi .
\end{aligned}
$$

In (B2), the sign of the kernel is determined by the cosine function since the Gauss hypergeometric function is an even positive function with respect to $\phi$. This argument allows demonstrating that (B2) is equal to zero. The integral in (B3) needs, however, to be calculated to derive the analytical expression of $N_{c}$. By introducing the change of variable $x=\cos ^{2}(\phi)$, (B3) results in

$$
\begin{aligned}
N_{c}=\frac{\Gamma(n+1 / 2)}{\sqrt{\pi} \Gamma(n)} & \left(1-|\rho|^{2}\right)^{n}|\rho| \\
& \times \int_{0}^{1} x^{\frac{1}{2}}(1-x)^{-\frac{1}{2}}\left(1-|\rho|^{2} x\right)^{-n-\frac{1}{2}} d x .
\end{aligned}
$$


By using 3.197(3) in [34, p. 314], the previous integral results in

$$
N_{c}=\frac{\Gamma(n+1 / 2) \Gamma(3 / 2)}{\Gamma(n)}\left(1-|\rho|^{2}\right)^{n}|\rho|_{2} F_{1}\left(n+\frac{1}{2}, \frac{3}{2} ; 2 ;|\rho|^{2}\right)
$$

which can be further simplified using the following identity among Gauss hypergeometric functions:

$$
{ }_{2} F_{1}(a, b ; c ; z)=(1-z)^{c-b-a} F_{1}(c-a, c-b ; c ; z) .
$$

Introducing (B6) into (B5), the final expression of the parameter $N_{c}(20)$ is obtained.

The parameter $N_{s}$, defined as

$$
N_{s}=\int_{-\pi}^{\pi} \sin (\phi) p_{\phi}(\phi) d \phi
$$

is obtained similarly. As in the case of (B2), it is possible to observe that $p_{\phi}(\phi)$ is an even positive function with respect to $\phi$. Since the sine function determines the sign of the integral kernel of (B7), it is straightforward to demonstrate that $N_{s}=0$.

\section{APPENDIX C \\ ASYMPTOTIC CHARACTERIZATION OF $N_{c}$}

Given (20), the following limit is analyzed:

$$
\lim _{n \rightarrow \infty} \frac{\Gamma(n+1 / 2) \Gamma(3 / 2)}{\Gamma(n)}|\rho|_{2} F_{1}\left(\frac{3}{2}-n, \frac{1}{2} ; 2 ;|\rho|^{2}\right) .
$$

Taking into account the asymptotic behavior of the ratio of Gamma functions expressed in (A2)

$$
\frac{\Gamma(n+1 / 2)}{\Gamma(n)}=\sqrt{n}
$$

for a large $n$. The asymptotic analysis of the Gauss hypergeometric function in (C1) is based on the results presented by Temme [41], who considers the asymptotic expansion of a particular family biorthogonal polynomials pairs, denoted by $\left\{P_{n}, Q_{n}\right\}$ on the unit circle. In particular, the polynomial $P_{n}$ presents the analytical expression

$$
P_{n}(z ; \alpha, \beta)={ }_{2} F_{1}(-n, \alpha+\beta+1 ; 2 \alpha+1 ; 1-z) .
$$

Under the conditions that $\alpha+\beta>1$, the parameter $z$ ranges in compact subsets of $\mathbb{C} /\{0\}$ and $(n+1) \ln \left(1-|\rho|^{2}\right)<0$, where the identity $1-|\rho|^{2}=z$ has been considered, (C3) presents the following nonuniform expansion:

$$
P_{n}(z ; \alpha, \beta)=\frac{\Gamma(2 \alpha+1)}{\Gamma(\alpha-\beta)}[(n+1)(1-z)]^{-\alpha-\beta-1}, \quad n \rightarrow \infty .
$$

As a result, if (C2) and (C4) are considered in (C1), the limit presented in (21) results. Due to the limitations imposed by (C4), the previous limit is not valid when $|\rho|=0$. Nevertheless, the analysis of the point $|\rho|=0$ results straightforward from (C1). Taking into account that ${ }_{2} F_{1}(3 / 2-n, 1 / 2 ; 2 ; 0)=1$

$$
\lim _{\substack{n \rightarrow \infty \\|\rho|=0}} \frac{\Gamma(n+1 / 2) \Gamma(3 / 2)}{\Gamma(n)}|\rho|_{2} F_{1}\left(\frac{3}{2}-n, \frac{1}{2} ; 2 ;|\rho|^{2}\right)=0 .
$$

\section{APPENDIX D}

$$
\text { EXPRESSIONS OF } \sigma_{\nu_{1}^{\prime}}^{2} \text { AND } \sigma_{\nu_{2}^{\prime}}^{2}
$$

Since the variance $\sigma_{\nu_{1}^{\prime}}^{2}$ can be expressed as follows:

$$
\sigma_{\nu_{1}^{\prime}}^{2}=E\left\{\left(\cos (\phi)-N_{c}\right)^{2}\right\}
$$

its analytical expression must obtained by means of the following integral:

$$
\int_{-\pi}^{\pi} \cos ^{2}(\phi) p_{\phi}(\phi) d \phi
$$

which results in the following two integrals:

$$
\begin{aligned}
& \frac{\Gamma(n+1 / 2)}{2 \sqrt{\pi} \Gamma(n)}\left(1-|\rho|^{2}\right)^{n}|\rho| \int_{-\pi}^{\pi} \frac{\cos ^{3}(\phi)}{\left(1-|\rho|^{2} \cos ^{2}\right)^{n+\frac{1}{2}}} d \phi \\
& \frac{1}{2 \pi}\left(1-|\rho|^{2}\right)^{n} \int_{-\pi}^{\pi} \cos ^{2}(\phi)_{2} F_{1}\left(n, 1 ; \frac{1}{2} ;|\rho|^{2} \cos ^{2}(\phi)\right) d \phi .
\end{aligned}
$$

Using the same arguments employed in Appendix B, it is possible to observe that (D3) is zero. The integral in (D4) is solved by means of 7.512(12) in [34, p. 807]. Then, by introducing the solution of (D4) and the expression of $N_{c}(20)$ into (D1), the variance $\sigma_{\nu_{1}^{\prime}}^{2}$ presents the expression detailed in (24). The variance $\sigma_{\nu_{2}^{\prime}}^{2}$ is obtained by means of the integral

$$
\sigma_{\nu_{2}^{\prime}}^{2}=\int_{-\pi}^{\pi} \sin ^{2}(\phi) p_{\phi}(\phi) d \phi
$$

which, when solved using the same procedure as in (D4), results into the expression presented by (25).

\section{APPENDIX E}

$$
\text { ASYMPTOTIC CHARACTERIZATION OF } \sigma_{\nu_{1}^{\prime}}^{2} \text { AND } \sigma_{\nu_{2}^{\prime}}^{2}
$$

In this case, the problem to tackle is to determine the asymptotics of the variances $\sigma_{\nu_{1}^{\prime}}^{2}$ and $\sigma_{\nu_{2}^{\prime}}^{2}$. As stated, a direct evaluation of the exact expressions of these parameters, (24) and (25), is quite difficult due to the extreme complexity of the process. Therefore, the process used to determine the asymptotics of $\sigma_{\nu_{1}^{\prime}}^{2}$ and $\sigma_{\nu_{2}^{\prime}}^{2}$ shall be based on considering their definitions on the basis of the asymptotic distribution of the Hermitian product phase difference presented in (15).

The asymptotic variance $\sigma_{\nu_{1}^{\prime}}^{2}$ is derived as defined in (D2), where the asymptotic pdf in (15) is considered. The integral 


$$
{ }_{2} F_{1}\left(n+\frac{1}{2}, 2 ; \frac{5}{2} ;|\rho|^{2}\right)=\sqrt{n}|\rho|\left(1-|\rho|^{2}\right)^{n}\left\{\frac{\sqrt{\pi}}{|\rho|^{4} n(n-1)}{ }_{2} F_{1}\left(2, \frac{1}{2} ; n+1 ; 1-\frac{1}{|\rho|^{2}}\right)+\frac{1}{\sqrt{n}|\rho|\left(1-|\rho|^{2}\right)^{n}}\left(1-\frac{1}{2 n|\rho|^{2}}\right)\right\}
$$

is solved by means of 3.197(3) by Gradshteyn and Ryzhik [34, p. 314], giving as a result

$$
\begin{aligned}
E\left\{\cos ^{2}(\phi)\right\} & =\frac{\sqrt{n}|\rho|}{\sqrt{\pi}}\left(1-|\rho|^{2}\right)^{n} \frac{\Gamma\left(\frac{1}{2}\right)}{\Gamma\left(\frac{5}{2}\right)} \\
& \times{ }_{2} F_{1}\left(n+\frac{1}{2}, 2 ; \frac{5}{2} ;|\rho|^{2}\right), \quad n \rightarrow \infty .
\end{aligned}
$$

By comparing (E1) with the real expression in (15), one can observe that it has been simplified in the sense that the former depends only on a Gauss hypergeometric function. Consequently, the next step is to study the asymptotics of the Gauss hypergeometric function in (E1). In this case, the transformation formula of the hypergeometric functions in (A5) is again considered. After some simplifications, it is possible to write (E2), as shown at the top of the page, where the definition of the Gauss hypergeometric function has been considered to simplify the second additive term. As observed, the asymptotics of (E2) is determined by this second additive term, since as observed, the first one is of the order $1 / n^{3 / 2}$. As a result

$$
E\left\{\cos ^{2}(\phi)\right\}=1-\frac{1}{2 n|\rho|^{2}}, \quad n \rightarrow \infty .
$$

Assuming the asymptotic behavior of the parameter $N_{c}$ and (E3), one can easily obtain the limit in (26).

The asymptotics of $\sigma_{\nu_{2}^{\prime}}^{2}$ are obtained by the same procedure as $\sigma_{\nu_{1}^{\prime}}^{2}$. The solution of the integral in (D5), by considering (15), is

$$
\begin{aligned}
E\left\{\sin ^{2}(\phi)\right\} & =\frac{\sqrt{n}|\rho|}{\sqrt{\pi}}\left(1-|\rho|^{2}\right)^{n} \frac{\Gamma\left(\frac{3}{2}\right)}{\Gamma\left(\frac{5}{2}\right)} \\
& \times{ }_{2} F_{1}\left(n+\frac{1}{2}, 1 ; \frac{5}{2} ;|\rho|^{2}\right), \quad n \rightarrow \infty .
\end{aligned}
$$

As observed, there are only subtle differences with respect to (E1), which, on the contrary, change completely the asymptotic behavior. Using the same transformation formula of hypergeometric functions in (A5), one gets

$$
\begin{aligned}
{ }_{2} F_{1}( & \left.n+\frac{1}{2}, 1 ; \frac{5}{2} ;|\rho|^{2}\right)=\frac{|\rho|}{\sqrt{\pi}} \Gamma\left(\frac{3}{2}\right)\left\{-\frac{\left(1-|\rho|^{2}\right)^{n}}{\Gamma\left(\frac{3}{2}\right) \sqrt{n}|\rho|^{2}}\right. \\
& \left.\times{ }_{2} F_{1}\left(1,-\frac{1}{2} ; n ; 1-\frac{1}{|\rho|^{2}}\right)+\frac{1}{2 n} \frac{\left(1-|\rho|^{2}\right)}{|\rho|^{2}}\right\}
\end{aligned}
$$

where, again, the asymptotic behavior is determined by the second additive term. In this case, the first additive term is proportional to $\left(1-|\rho|^{2}\right)^{n} / n^{1 / 2}$. Consequently

$$
E\left\{\sin ^{2}(\phi)\right\}=\frac{1}{2 n} \frac{\left(1-|\rho|^{2}\right)}{|\rho|^{2}}, \quad n \rightarrow \infty
$$

which easily show the limit detailed in (26).
In order to determine the asymptotic behavior of (24) and (25) in $|\rho|=0$, the following generalized power series of the generalized hypergeometric function is taken into account [40]:

$$
{ }_{3} F_{2}(a, b, c ; d, e ; z) \propto 1+O(z), \quad z \rightarrow 0 .
$$

As a result, a first-order approximation of $\sigma_{\nu_{1}^{\prime}}^{2}$ and $\sigma_{\nu_{2}^{\prime}}^{2}$ in the vicinity $|\rho|=0$ is obtained, as observed in (27).

\section{ACKNOWLEDGMENT}

The authors would like to thank DLR for providing the Traunstein dataset and J.-S. Lee and N. Temme for their fruitful comments.

\section{REFERENCES}

[1] J. C. Curlander and R. N. McDonough, Synthetic Aperture Radar: Systems and Signal Processing. New York: Wiley, 1991.

[2] J. W. Goodman, "Some fundamental properties of speckle," J. Opt. Soc. Amer., vol. 66, no. 11, pp. 1145-1149, Nov. 1976.

[3] _- Statistical Optics. New York: Wiley, 1984.

[4] J.-S. Lee, "Speckle analysis and smoothing of synthetic aperture radar images," Comput. Graph. Image Process., vol. 17, no. 1, pp. 24-32, Sep. 1981.

[5] A. Lopes, R. Touzi, and E. Nezry, "Adaptive speckle filters and scene heterogeneity," IEEE Trans. Geosci. Remote Sens., vol. 28, no. 6, pp. 992 1000, Nov. 1990.

[6] R. Bamler and P. Hartl, "Synthetic aperture radar interferometry," Inv. Probl., vol. 14, no. 4, pp. R1-R54, Aug. 1998.

[7] C. H. Gierull, C. Livingstone, "SAR-GMTI concept for RADARSAT-2," in The Applications of Space-Time Processing, R. Klemm, Ed. Stevenage, U.K.: IEE Press, 2004.

[8] F. T. Ulaby and C. Elachi, Radar Polarimetry for Geoscience Applications. Norwood, MA: Artech House, 1990.

[9] C. López-Martínez and X. Fàbregas, "Polarimetric SAR speckle noise model," IEEE Trans. Geosci. Remote Sens., vol. 41, no. 10, pp. 2232 2242, Oct. 2003.

[10] C. López-Martínez and E. Pottier, "Topography independent InSAR coherence estimation in a multiresolution scheme," in Proc. IGARSS, Seoul, Korea, Jul. 2005, pp. 2689-2692.

[11] _ _ "A framework for the analysis of speckle noise effects in multidimensional SAR imagery," in Proc. IGARSS, Seoul, Korea, Jul. 2005, pp. 4840-4843.

[12] C. López-Martínez, E. Pottier, and S. R. Cloude, "Statistical assessment of eigenvector-based target decomposition theorems in radar polarimetry," IEEE Trans. Geosci. Remote Sens., vol. 43, no. 9, pp. 2058-2074, Sep. 2005.

[13] N. R. Goodman, "Statistical analysis based on a certain multivariate complex Gaussian distribution (an introduction)," Ann. Math. Stat., vol. 34, no. 1, pp. 152-177, Mar. 1963.

[14] S. R. Cloude and E. Pottier, "A review of target decomposition theorems in radar polarimetry," IEEE Trans. Geosci. Remote Sens., vol. 34, no. 2, pp. 498-518, Mar. 1996.

[15] A. Reigber and A. Moreira, "First demonstration of airborne SAR tomography using multibaseline L-band data," IEEE Trans. Geosci. Remote Sens., vol. 38, no. 5, pp. 2142-2152, Sep. 2000.

[16] K. Sarabandi, " $\Delta k$-radar equivalent of interferometric's SAR: A theoretical study for determination of vegetation height," IEEE Trans. Geosci. Remote Sens., vol. 35, no. 5, pp. 1267-1275, Sep. 1997.

[17] J.-S. Lee, M. R. Grunes, and S. A. Mango, "Speckle reduction in multipolarization, multifrequency SAR imagery," IEEE Trans. Geosci. Remote Sens., vol. 29, no. 4, pp. 535-544, Jul. 1991.

[18] R. M. A. Azzam and N. M. Bashara, Ellipsometry and Polarized Light. Amsterdam, The Netherlands: Elsevier, 1987. 
[19] S. N. Madsen, "Modelling, analysis, and applications related to synthetic aperture radar data," Ph.D. dissertation, Tech. Univ. Denmark, Lyngby, Denmark, Nov. 1986.

[20] R. L. Mitchel, "Models of extended targers and their coherence radar images," Proc. IEEE, vol. 62, no. 6, pp. 754-758, Jun. 1974.

[21] C. Oliver and S. Quegan, Understanding Synthetic Aperture Radar Images. Boston, MA: Artech House, 1998.

[22] R. J. Q. Tough, D. Blacknell, and S. Quegan, "A statistical description of polarimetric and interferometric synthetic aperture radar data," Proc. R. Soc. Lond. A, Math. Phys. Sci., vol. 449, no. 1937, pp. 567-589, Jun. 1995.

[23] J.-S. Lee, K. W. Hoppel, and S. A. Mango, "Intensity and phase statistics of multilook polarimetric interferometric SAR imagery," IEEE Trans. Geosci. Remote Sens., vol. 32, no. 5, pp. 1017-1028, Sep. 1994.

[24] A. Lopés and F. Sery, "Optimal speckle reduction for the product model in multilook polarimetric SAR imagery and the Wishart distribution," IEEE Trans. Geosci. Remote Sens., vol. 35, no. 3, pp. 632-647, May 1997.

[25] J. Shou and H. Skriver, "Restoration of polarimetric SAR images using simulated annealing," IEEE Trans. Geosci. Remote Sens., vol. 39, no. 9, pp. 2005-2016, Sep. 2001.

[26] J.-S. Lee, M. R. Grunes, and G. de Grandi, "Polarimetric SAR speckle filtering and its impact for classification," IEEE Trans. Geosci. Remote Sens., vol. 35, no. 5, pp. 2363-2373, Sep. 1999.

[27] L. Ferro-Famil, E. Pottier, and J.-S. Lee, "Unsupervised classification of multifrequency and fully polarimetric SAR images based on the H/A/Alpha-Wishart classifier," IEEE Trans. Geosci. Remote Sens., vol. 39, no. 11, pp. 2332-2342, Nov. 2001.

[28] J.-S. Lee, M. R. Grunes, E. Portier, and L. Ferro-Famil, "Unsupervised terrain classification preserving polarimetric scattering characteristics," IEEE Trans. Geosci. Remote Sens., vol. 42, no. 4, pp. 722-731, Apr. 2004.

[29] J.-S. Lee, M. R. Grunes, T. L. Ainsworth, L. J. Du, D. L. Schuler, and S. R. Cloude, "Unsupervised classification using polarimetric decomposition and the complex Wishart classifier," IEEE Trans. Geosci. Remote Sens., vol. 37, no. 5, pp. 2249-2258, Sep. 1999.

[30] I. Reed, "On a moment theorem for complex Gaussian processes," IEEE Trans. Inf. Theory, vol. IT-8, no. 3, pp. 194-195, Apr. 1962

[31] G. Letac and H. Massam, "All invariant moments of the Wishart distribution," Scand. J. Stat., vol. 31, no. 2, pp. 295-318, Jun. 2004.

[32] H. Krim, P. Forster, and J. G. Proakis, "Operator approach to performance analysis of root-MUSIC and root-min-norm," IEEE Trans. Signal Process., vol. 40, no. 7, pp. 1687-1696, Jul. 1992.

[33] K. Sarabandi, "Derivation of phase statistics from the Müeller matrix," Radio Sci., vol. 27, no. 5, pp. 553-560, Sep. 1992.

[34] I. S. Gradshteyn and I. M. Ryzhik, Table of Integrals, Series and Products, 6th ed. San Diego, CA: Academic, 2000.

[35] M. Abramowitz and I. A. Stegun, Handbook of Mathematical Functions, 9th ed. New York: Dover, 1972.

[36] J.-S. Lee, K. P. Papathanassiou, T. L. Ainsworth et al., "A new technique for noise filtering of SAR interferometric phase images," IEEE Trans. Geosci. Remote Sens., vol. 36, no. 5, pp. 1456-1465, Sep. 1998.

[37] N. M. Temme, "Large parameter cases of the Gauss hypergeometric function," J. Comput. Appl. Math., vol. 153, no. 1/2, pp. 441-462, Apr. 2003.

[38] C. López-Martínez and X. Fàbregas, "Modelling and reduction of SAR interferometric phase noise in the wavelet domain," IEEE Trans. Geosci. Remote Sens., vol. 40, no. 12, pp. 2553-2566, Dec. 2002.

[39] Y. L. Luke, The Special Functions and Their Approximations, vol. I. New York: Academic, 1969.

[40] E. W. Weisstein, "Gamma function," in MathWorld-A Wolfram Web Resource. [Online]. Available: http://mathworld.wolfram.com/ GammaFunction.html

[41] N. M. Temme, "Uniform asymptotic expansion for a class of polynomials biorthogonal on the unit circle," Constr. Approx., vol. 2, no. 1, pp. 369-376, Dec. 1986.

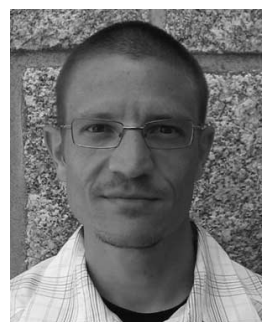

Carlos López-Martínez (S'96-M'04) received the M.Sc. degree in electrical engineering and the Ph.D. degree from the Technical University of Catalonia (UPC), Barcelona, Spain, in 1999 and 2003, respectively.

In 1999, he joined the Signal Theory and Communications Department, UPC, where he developed his Ph.D. thesis, which focused on multidimensional speckle noise modeling and reduction. From October 2000 until March 2002, he was with the Frequency and Radar Systems Department (HR), German Aerospace Center, Oberpfaffenhofen. Since June 2003, he has been with the Image and Remote Sensing Group-SAPHIR Team, Institute of Electronics and Telecommunications of Rennes (IETR-CNRS UMR 6164). His research interests include synthetic aperture radar and multidimensional SAR, radar polarimetry, digital signal processing, estimation theory, and harmonic analysis.

Dr. López-Martínez received the Student Prize Paper Award at the EUSAR 2002 Conference, Cologne, Germany.

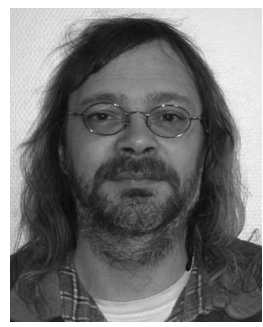

Eric Pottier (M'95) received the M.Sc. and Ph.D. degrees in signal processing and telecommunication from the University of Rennes 1, Rennes, France, and the Habilitation from the University of Nantes, Nantes, France, in 1987, 1990, and 1998, respectively.

From 1988 to 1999, he was an Associate Professor with the IRESTE-University of Nantes, Nantes, France, where he was the Head of the Polarimetry Group of the Electronic and Informatic Systems Laboratory. Since 1999, he has been a Full Professor with the University of Rennes 1, France, where he is currently the Deputy Director of the Institute of Electronics and Telecommunications of Rennes (IETR - CNRS UMR 6164) and also the Head of the Image and Remote Sensing Group-SAPHIR Team. Since 1989, he has been supervising more than 50 research students to graduation (M.Sc. and Ph.D.) in radar polarimetry, covering areas from theory to remote sensing applications. His current activities of research and education are centered in the topics of analog electronics, microwave theory, and radar imaging with emphasis in radar polarimetry. His research covers a wide spectrum of areas from radar image processing (SAR and inverse SAR), polarimetric scattering modeling, supervised/unsupervised polarimetric segmentation, and classification to fundamentals and basic theory of polarimetry. He has six publications in books, 23 papers in refereed journals, and more than 160 papers in conference and symposium proceedings. He has presented advance courses and seminars on radar polarimetry to a wide range of organization [German Aerospace Center (DLR), National Space Development Agency of Japan (NASDA), Joint Research Center (JRC), Remote Sensing Technology Center of Japan (RESTEC), ISAP2000, IGARSS03, EUSAR04, North Atlantic Treaty Organisation (NATO), PolInSAR05 and IGARSS05].

Dr. Pottier has chaired and organized 21 sessions in international conferences and was a member of the Technical and Scientific Committees of 17 international symposium or conferences. He has been invited to present 21 presentations in international conferences and 14 in national conferences. He received the Best Paper Award during EUSAR2000 for his research activities, coauthored with J.-S. Lee (US Navy/NRL), in the topic of polarimetric unsupervised segmentation of PolSAR data. 